\title{
Master Schedule for CY-1980 Hanford Environmental Surveillance Routine Program
}

\author{
P. J. Blumer \\ J. R. Houston \\ P. A. Eddy
}

December 1979

Prepared for the U.S. Department of Energy under Contract EY-76-C-06-1830

Pacific Northwest Laboratory

Operated for the U.S. Department of Energy by Battelle Memorial Institute 
NOTICE

This report was prepared as an account of work sponsored by the United States Government. Neither the United States nor the Department of Energy, nor any of their empioyees, nor any of their contractors, subcontractors, or their employees, makes any warranty, express or implied, or assumes any legal liability or responsibility for the accuracy, completeness or usefulness of any information, apparatus, product or process disclosed, or represents that its use would not infringe privately owned rights.

The views, opinions and conclusions contained in this report are those of the contractor and do not necessarily represent those of the United States Government or the United States Department of Energy.

\author{
PACIFIC NORTHWEST LABORATORY \\ operated by \\ BATTELLE \\ for the \\ UNITED STATES DEPARTMENT OF ENERGY \\ Under Contract EY-76-C-06-1830
}

\begin{tabular}{|c|c|c|}
\hline \multicolumn{3}{|c|}{$\begin{array}{c}\text { Printed in the United States of An } \\
\text { Available from } \\
\text { National Technical Information St } \\
\text { United States Department of Com } \\
5285 \text { Port Royal Road } \\
\text { Springfield, Virginia 22151 }\end{array}$} \\
\hline Price: & Printed Copy S & - Microf \\
\hline & -Pages & $\begin{array}{c}\text { NTIS } \\
\text { Selling Price }\end{array}$ \\
\hline & $001-025$ & $\$ 4.00$ \\
\hline & $026-050$ & $\$ 4.50$ \\
\hline & $051-075$ & $\$ 5.25$ \\
\hline & $076-100$ & $\$ 6.00$ \\
\hline & $101-125$ & $\$ 6.50$ \\
\hline & $126-150$ & 57.25 \\
\hline & $151-175$ & $\$ 8.00$ \\
\hline & $176-200$ & $\$ 9,00$ \\
\hline & $201-225$ & $\$ 9.25$ \\
\hline & $226-250$ & $\$ 9.50$ \\
\hline & $251-275$ & $\$ 10.75$ \\
\hline & $276-300$ & $\$ 11.00$ \\
\hline
\end{tabular}


PNL-3222

UC -41

\section{4}

MASTER SCHEDULE FOR CY-1980

HANFORD ENVIRONMENTAL SURVEILLANCE

ROUTINE PROGRAM

P. J. Blumer

J. R. Houston

P. A. Eddy

December 1979

Prepared for

the U.S. Department of Energy

under Contract EY-76-C-06-1830

Pacific Northwest Laboratory

Richland, Washington 99352 
i 
CONTENTS

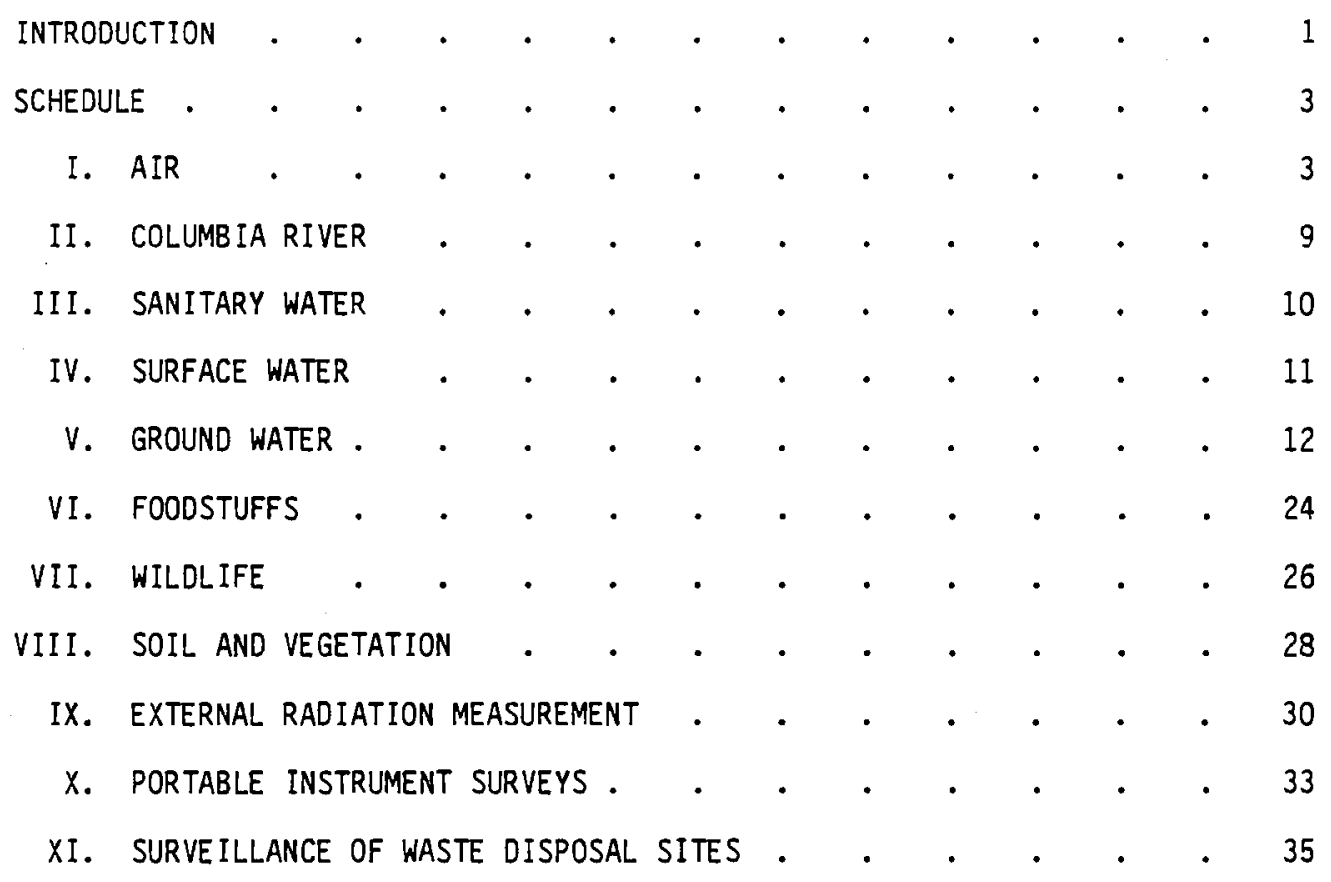


!

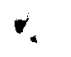

. 


\section{INTRODUCTION}

This report provides the current schedule of data collection for the routine environmental surveillance program at the Hanford Site, as conducted by the Environmental Evaluations Section of the Pacific Northwest Laboratory(a) for the Department of Energy. Questions about specific entries should be referred to the authors since modifications to the schedule are made during the year and special areas of study, usually of short duration, are not scheduled.

The environmental surveillance program objectives are to evaluate the levels of radioactive and nonradioactive pollutants in the Hanford environs, as required in Manual Chapter 0513, and to monitor Hanford operations for compliance with applicable environmental criteria given in Manual Chapter 0524 and Washington State Water Quality Standards. Air quality data are obtained in a separate program administered by the Hanford Environmental Health Foundation. The collection schedule for potable water is shown but it is not part of the routine environmental surveillance program. Water quality data for Hanford Site potable water systems are published each year by the Hanford Environmental Health Foundation.

The data collected are available in routine reports issued by the Environmental Evaluations staff. Groundwater data and evaluations are reported in the series, "Radiological Status of the Groundwater Beneath the Hanford Project for...," the latest issue being PNL-2899 for CY-1978. Data from locations within the plant boundaries are presented in the annual "Environmental Status of the Hanford Site for..." report series, the most recent report being PNL-2933 for 1978. Data from offsite locations are presented in the annual "Environmental Surveillance at Hanford for..." series of reports, the latest being PNL-2932 for 1978.

(a) Operated by Battelle Memorial Institute. 
Frequency Symbols Used

$\begin{array}{lc}\text { D - Daily } & \text { M Comp. - Monthly Composite } \\ \text { W - Weekly } & \text { Q - Quarterly } \\ \text { BW - Biweekly (every 2 wk) } & \text { A - Annually } \\ \text { M - Monthly } & \text { SA - Semiannually } \\ \text { BM - Bimonthly (every 2 mo) } & \text { NRA - Not Routinely Analyzed }\end{array}$


I. AIR

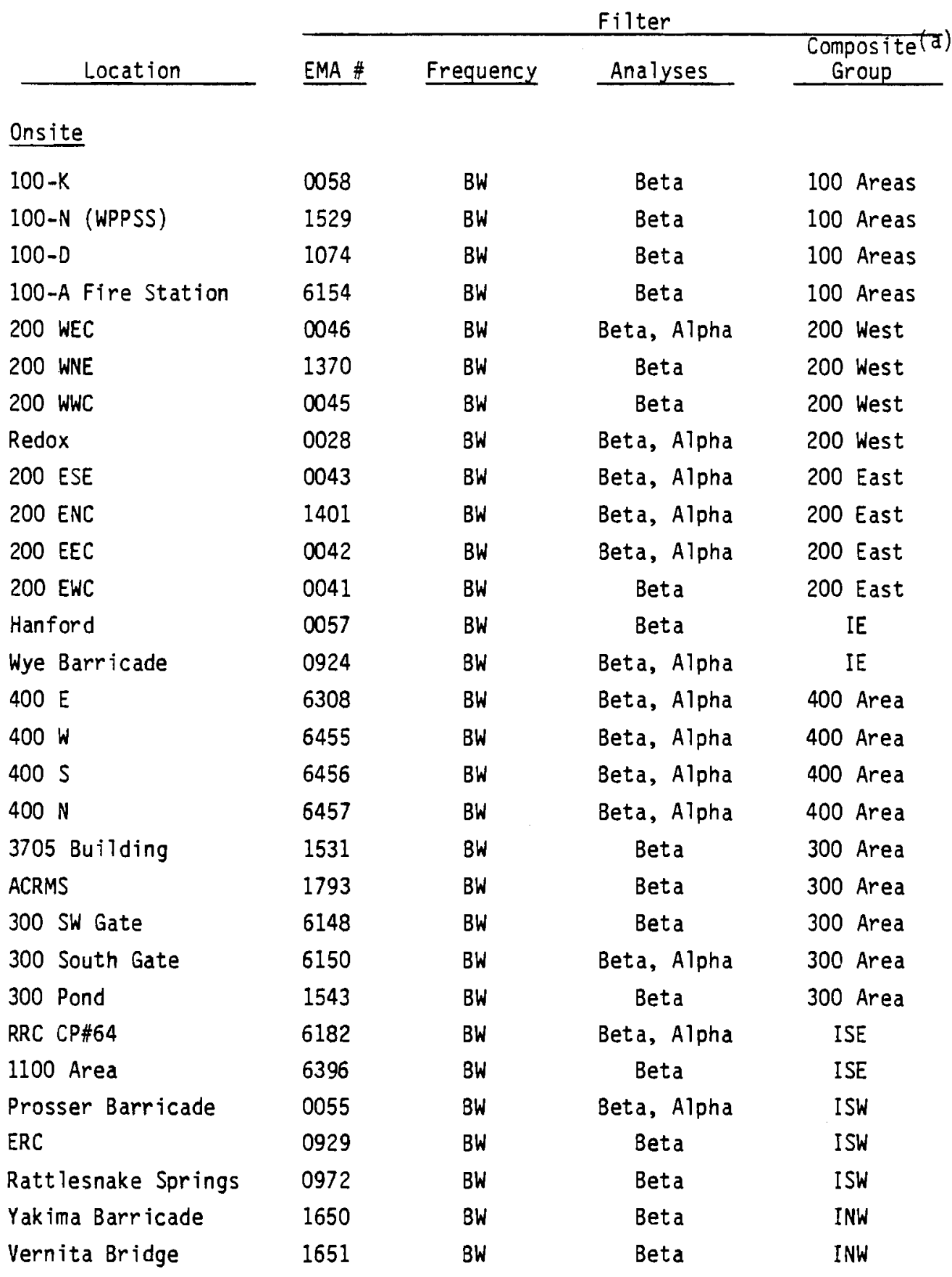

(a) Composite group for filter analyses shown on page 7 . 
I. AIR (contd)

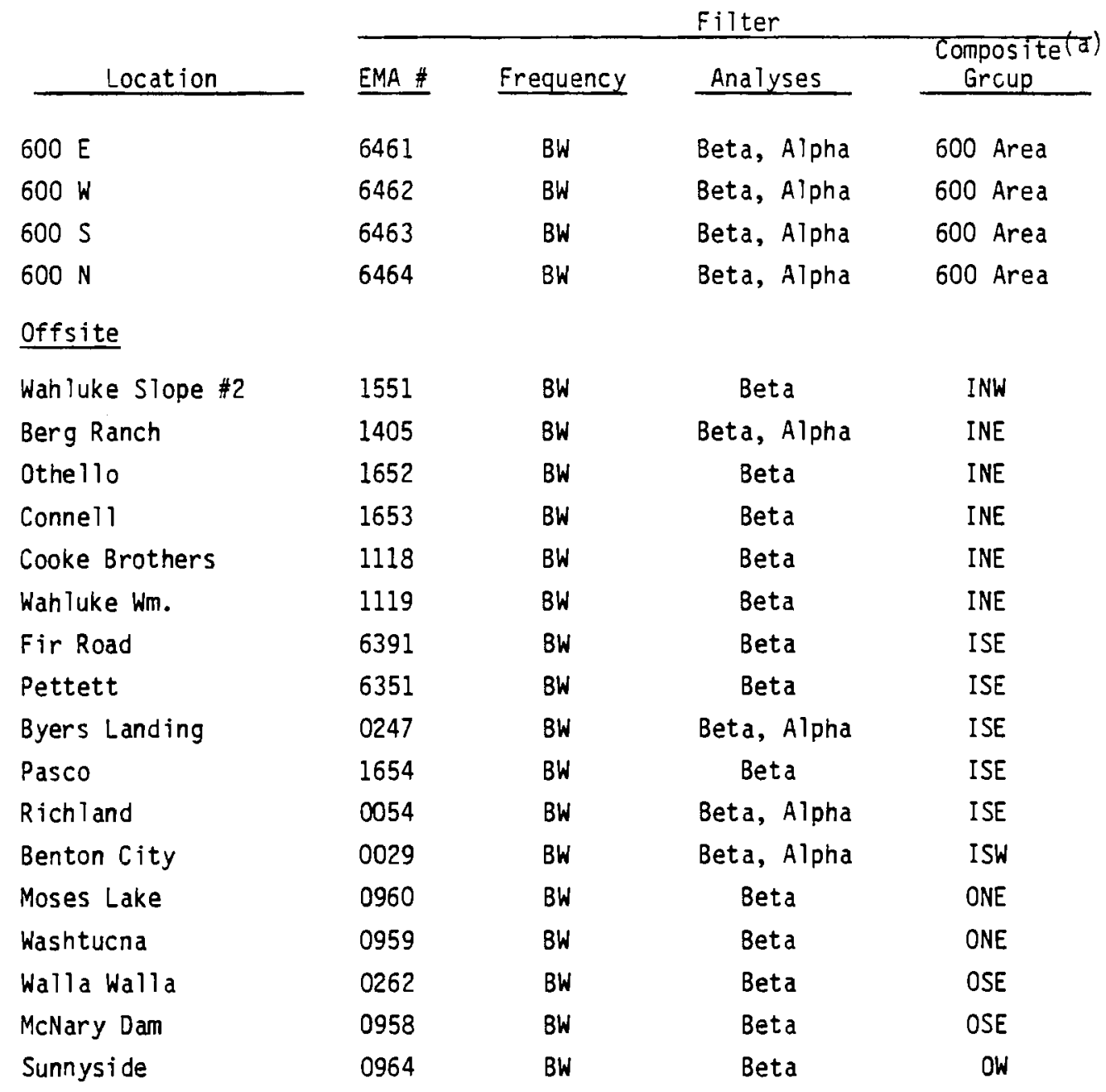

(a) Composite group for filter analyses shown on page 7 . 
I. $\quad \underline{A R}$ (contd)

Charcoal Cartridge Tritium Cartridge

EMA \# Frequency Analyses EMA \# Frequency Analyses

Onsite

\begin{tabular}{|c|c|c|c|c|c|c|}
\hline $100-K$ & 1581 & M & NRA & & & \\
\hline 100-N (WPPSS) & 1661 & M & NRA & & & \\
\hline $100-0$ & 1582 & $B W$ & ${ }^{131_{I}}$ & 6335 & BW & HTO \\
\hline 100-A Fire Station & 6155 & M & NRA & & & \\
\hline 200 WEC & 1662 & M & NRA & & & \\
\hline 200 WNE & 6272 & M & NRA & & & \\
\hline $200 W W C$ & 6152 & M & NRA & & & \\
\hline Redox & 1663 & $M$ & NRA & & & \\
\hline 200 ESE & 1664 & BW & ${ }^{131}$ & 6201 & $\mathrm{BW}$ & HTO \\
\hline 200 ENC & 1665 & M & NRA & & & \\
\hline 200 EEC & 1361 & M & NRA & & & \\
\hline 200 EWC & 6273 & M & NRA & & & \\
\hline Hanford & 1666 & M & NRA & & & \\
\hline Wye Barricade & 1584 & M & NRA & & & \\
\hline $400 \mathrm{E}$ & 6309 & $\mathrm{BW}$ & 131 & 6428 & $B W$ & HTO \\
\hline $400 \mathrm{~W}$ & 6458 & $8 W$ & $131_{I}$ & & & \\
\hline $400 \mathrm{~s}$ & 6459 & $B W$ & $131_{I}$ & & & \\
\hline $400 \mathrm{~N}$ & 6460 & BW & ${ }^{131} 1_{I}$ & & & \\
\hline 3705 Building & 1669 & $M$ & NRA & & & \\
\hline ACRMS & 1795 & M & NRA & & & \\
\hline 300 SW Gate & 6149 & $B W$ & ${ }^{131} I$ & & & \\
\hline 300 South Gate & 6151 & M & NRA & & & \\
\hline 300 Pond & 6239 & $M$ & NRA & & & \\
\hline RRC CP\#64 & 6183 & M & NRA & & & \\
\hline 1100 Area & 6397 & M & NRA & & & \\
\hline Prosser Barricade & 6248 & M & NRA & & & \\
\hline ERC & 1585 & M & NRA & & & \\
\hline Rattlesnake Springs & 1586 & M & NRA & & & \\
\hline Yakima Barricade & 1667 & M & NRA & & & \\
\hline Vernita Bridge & 1668 & M & NRA & & & \\
\hline \multicolumn{7}{|l|}{ Offsite } \\
\hline Wahluke Slope \#2 & 1671 & M & NRA & & & \\
\hline Berg Ranch & 1672 & M & NRA & & & \\
\hline Othe 110 & 1673 & $M$ & NRA & & & \\
\hline
\end{tabular}


I. AIR (contd)

\begin{tabular}{|c|c|c|c|c|c|c|}
\hline \multirow[b]{2}{*}{ Location } & \multicolumn{3}{|c|}{ Charcoal Cartridge } & \multicolumn{3}{|c|}{ Tritium Cartridge } \\
\hline & EMA \# & Frequency & Analyses & EMA \# & Frequency & Analyses \\
\hline Conne 11 & 1674 & M & NRA & & & \\
\hline Cooke Brothers & 1675 & M & NRA & & & \\
\hline Wahluke Wm. & 1676 & M & NRA & & & \\
\hline Fir Road & 6392 & $B W$ & 131 & 6393 & $B W$ & HTO \\
\hline Pettett & 6352 & BW & ${ }^{131}$ I & & & \\
\hline Byers Landing & 0246 & $B W$ & ${ }^{131_{I}}$ & & & \\
\hline Pasco & 1678 & M & NRA & & & \\
\hline Rich Tand & 0231 & $B W$ & ${ }^{131} \mathrm{I}$ & 6207 & BW & HTO \\
\hline Benton City & 1670 & $\mathrm{BW}$ & ${ }^{131}$ I & 6411 & $\mathrm{BW}$ & HTO \\
\hline Moses Lake & 1682 & M & NRA & & & \\
\hline Washtucna & 1683 & M & NRA & & & \\
\hline Walla Walla & 0261 & M & NRA & & & \\
\hline McNary Dam & 1684 & M & NRA & & & \\
\hline Sunnyside & 1680 & $\mathrm{BW}$ & ${ }^{131} 1_{I}$ & & & \\
\hline
\end{tabular}


I. AIR (contd)

\begin{tabular}{|c|c|c|c|}
\hline Composite Group & EMA \# & Frequency & Anaiyses \\
\hline $\begin{array}{l}200 \text { East } \\
200 \text { ENC } \\
200 \text { EWC } \\
200 \text { ESE } \\
200 \text { EEC }\end{array}$ & 1749 & $\begin{array}{l}M \\
0\end{array}$ & $\begin{array}{l}\text { Gamma scan } \\
{ }^{90} \mathrm{Sr} \text {, Pu-total }\end{array}$ \\
\hline $\begin{array}{l}200 \text { West } \\
200 \text { WEC } \\
200 \text { WWC } \\
\text { Redox } \\
200 \text { WNE }\end{array}$ & 1751 & $\begin{array}{l}M \\
Q\end{array}$ & $\begin{array}{l}\text { Garma scan } \\
{ }^{90} \mathrm{Sr} \text {, Pu-tota } 1\end{array}$ \\
\hline $\begin{array}{l}300 \text { Area } \\
300 \text { Area, } 3705 \text { Building } \\
\text { ACRMS } \\
300 \text { South Gate } \\
300 \text { Southwest Gate } \\
300 \text { Pond }\end{array}$ & 1752 & $\begin{array}{l}M \\
Q\end{array}$ & $\begin{array}{l}\text { Gamma scan } \\
90 \text { Sr, Pu-tota } 1\end{array}$ \\
\hline $\begin{array}{l}400 \text { Area } \\
400 \mathrm{E} \\
400 \mathrm{~W} \\
400 \mathrm{~S} \\
400 \mathrm{~N}\end{array}$ & 6465 & $\begin{array}{l}M \\
Q\end{array}$ & $\begin{array}{l}\text { Gamma scan } \\
90 \text { Sr, Pu-tota } 1\end{array}$ \\
\hline $\begin{array}{l}100 \text { Areas } \\
100 \text { Area Fire Station } \\
100-\mathrm{K} \\
100-\mathrm{N} \\
100-\mathrm{D}\end{array}$ & 1753 & $\begin{array}{l}M \\
Q\end{array}$ & $\begin{array}{l}\text { Garmma scan } \\
{ }^{90} \mathrm{Sr} \text {, Pu-tota } 1\end{array}$ \\
\hline $\begin{array}{l}600 \text { Area } \\
600 \mathrm{E} \\
600 \mathrm{~W} \\
600 \mathrm{~S} \\
600 \mathrm{~N}\end{array}$ & 6466 & $\begin{array}{l}M \\
Q\end{array}$ & $\begin{array}{l}\text { Garmma scan } \\
90 \mathrm{Sr}, \text { Pu-total } 1\end{array}$ \\
\hline $\begin{array}{l}\text { Inner Eastern Quadrant } \\
\text { Hanford }\end{array}$ & 1754 & $\begin{array}{l}M \\
Q\end{array}$ & $\begin{array}{l}\text { Garmma scan } \\
90 \text { Sr, Pu-tota } 1\end{array}$ \\
\hline
\end{tabular}


I. AIR (contd)

\begin{tabular}{|c|c|c|c|}
\hline Composite Group & EMA \# & Frequency & Analyses \\
\hline $\begin{array}{l}\text { Inner Southeast Quadrant } \\
\text { RRC CP } \# 64 \\
1100 \text { Area } \\
\text { Richland } \\
\text { Pasco } \\
\text { Byers Landing } \\
\text { Fir Road } \\
\text { Pettett }\end{array}$ & 1745 & $\begin{array}{l}M \\
Q\end{array}$ & $\begin{array}{l}\text { Gamma scan } \\
90 \mathrm{Sr} \text {, Pu-total }\end{array}$ \\
\hline $\begin{array}{l}\text { Inner Southwest Quadrant } \\
\text { Ratt lesnake Springs } \\
\text { ERC } \\
\text { Benton City } \\
\text { Prosser Barricade }\end{array}$ & 1746 & $\begin{array}{l}M \\
Q\end{array}$ & $\begin{array}{l}\text { Gammla scan } \\
90_{\text {Sr, Pu-total }}\end{array}$ \\
\hline $\begin{array}{l}\text { Inner Northwest Quadrant } \\
\text { Yakima Barricade } \\
\text { Vernita } \\
\text { Wahluke \#2 }\end{array}$ & 1747 & $\begin{array}{l}M \\
Q\end{array}$ & $\begin{array}{l}\text { Gamma scan } \\
{ }^{90} \mathrm{Sr} \text {, Pu-total }\end{array}$ \\
\hline $\begin{array}{l}\text { Inner Northeast Quadrant } \\
\text { Othe } 110 \\
\text { Connell } \\
\text { Berg Ranch } \\
\text { Wahluke Watermaster } \\
\text { Cooke Brothers }\end{array}$ & 1743 & $\begin{array}{l}M \\
Q\end{array}$ & $\begin{array}{l}\text { Ganma scan } \\
{ }^{90} \mathrm{Sr} \text {, Pu-total }\end{array}$ \\
\hline $\begin{array}{l}\text { Outer Northeast Quadrant } \\
\text { Moses Lake } \\
\text { Washtucna }\end{array}$ & 1742 & $\begin{array}{l}M \\
Q\end{array}$ & $\begin{array}{l}\text { Gamma scan } \\
{ }^{90} \mathrm{Sr} \text {, Pu-total }\end{array}$ \\
\hline $\begin{array}{l}\text { Outer Southeast Quadrant } \\
\text { Walla Walla } \\
\text { McNary }\end{array}$ & 1744 & $\begin{array}{l}M \\
Q\end{array}$ & $\begin{array}{l}\text { Garma scan } \\
{ }^{90} \mathrm{Sr} \text {, Pu-total }\end{array}$ \\
\hline $\begin{array}{l}\text { Outer Western Quadrant } \\
\text { Sunnyside }\end{array}$ & 1748 & $\begin{array}{l}M \\
Q\end{array}$ & $\begin{array}{l}\text { Gamma scan } \\
90 \text { Sr, Pu-tota }]\end{array}$ \\
\hline
\end{tabular}


II. COLUMB IA RIVER

\begin{tabular}{|c|c|c|c|c|}
\hline Sample Type & Location & EMA \# & Frequency & Analyses \\
\hline \multirow[t]{4}{*}{ "Filter-Resin" } & $\begin{array}{l}\text { Priest Rapids Oam } \\
\text { Resin Column }\end{array}$ & 6394 & $\begin{array}{l}B W \\
Q\end{array}$ & $\begin{array}{l}\text { Gamma scan }\left({ }^{131} \mathrm{I}\right) \\
\mathrm{Pu},{ }^{129} \mathrm{I}\end{array}$ \\
\hline & Filters & 6395 & $\begin{array}{l}B W \\
Q\end{array}$ & $\begin{array}{l}\text { Gamma scan }\left({ }^{131} \mathrm{I}\right) \\
\mathrm{Pu},{ }^{129} \mathrm{I}\end{array}$ \\
\hline & $\begin{array}{l}300 \text { Area Forebay } \\
\text { Resin Column }\end{array}$ & 6384 & $\begin{array}{l}B W \\
Q\end{array}$ & $\begin{array}{l}\text { Gamma scan }\left({ }^{131} \mathrm{I}\right) \\
\mathrm{Pu},{ }^{129} \mathrm{I}\end{array}$ \\
\hline & Filters & 6385 & $\begin{array}{l}\text { BW } \\
Q\end{array}$ & $\begin{array}{l}\text { Gamma scan }\left({ }^{131} \mathrm{I}\right) \\
\text { Pu, }{ }^{129} \mathrm{I}\end{array}$ \\
\hline \multirow[t]{2}{*}{ Cumulative } & $100-B$ & 6156 & $\begin{array}{l}\text { M comp } \\
Q\end{array}$ & $\begin{array}{l}\text { Alpha, Beta, }{ }^{3} \mathrm{H} \text {, } \\
\text { Gamma scan, } U \\
{ }^{89} \mathrm{Sr},{ }^{90} \mathrm{Sr},{ }^{226} \mathrm{Ra} \text {, } \\
{ }^{22}{ }_{\mathrm{Ra}}\end{array}$ \\
\hline & Rich 1 and & 1000 & $\begin{array}{l}\text { M comp } \\
Q\end{array}$ & $\begin{array}{l}\text { Alpha, Beta, }{ }^{3} \mathrm{H} \text {, } \\
\text { Gamma scan, } U \\
{ }^{89} \mathrm{Sr},{ }^{90} \mathrm{Sr},{ }^{226} \mathrm{Ra} \text {, } \\
{ }^{228} \mathrm{R}_{\mathrm{Ra}}\end{array}$ \\
\hline \multirow[t]{3}{*}{ Grab } & Vernita & $\begin{array}{l}1204 \\
1616 \\
1373\end{array}$ & $\begin{array}{l}W \\
W \\
W *\end{array}$ & $\begin{array}{l}\mathrm{pH} \text {, Diss } \mathrm{O}_{2} \text {, Turbidity } \\
\mathrm{NO}_{3}^{-} \\
\text {Coliforms, Fecal coli- } \\
\text { forms, } 800\end{array}$ \\
\hline & Richland Forebay & $\begin{array}{l}1617 \\
1365\end{array}$ & $\begin{array}{l}W \\
M *\end{array}$ & $\begin{array}{l}\mathrm{NO}_{3}^{-} \\
\mathrm{Coliforms,} \mathrm{BOD} \text {, Fecal } \\
\text { coliforms }\end{array}$ \\
\hline & & 6429 & w & $\mathrm{pH}$, Diss $\mathrm{O}_{2}$, turbidity \\
\hline
\end{tabular}

* Performed by HEHF. 
III. SANITARY WATER

\begin{tabular}{|c|c|c|c|c|}
\hline Sample Type & Location & EMA \# & Frequency & Analyses \\
\hline \multirow[t]{6}{*}{ Cumulative } & Richland & 1002 & $W$ & Alpha, Beta \\
\hline & & & M comp & Gamma scan \\
\hline & & 1366 & $W *$ & $\mathrm{NO}_{3}$ \\
\hline & 300 Area & 1686 & $M$ comp & Alpha, Beta, Gamma scan \\
\hline & & 1724 & $w^{\star}$ & $\mathrm{NO}_{3}$ \\
\hline & FFTF & 6382 & $M$ comp & Beta, Alpha, ${ }^{3} \mathrm{H}$ \\
\hline \multirow[t]{10}{*}{ Grab } & Observatory & 6381 & $Q$ & Alpha, Beta \\
\hline & $100-0$ & 1219 & $Q$ & Alpha, Beta \\
\hline & 200 East & 6374 & $Q$ & Alpha, Beta \\
\hline & 200 West & 6375 & $Q$ & Alpha, Beta \\
\hline & 100 Area Fire & & & \\
\hline & Station & 6376 & $Q$ & Alpha, Beta \\
\hline & 200 Area Fire & & & \\
\hline & Station & 6377 & $Q$ & Alpha, Beta \\
\hline & 251 Building & 6378 & $Q$ & Alpha, Beta \\
\hline & Met Tower & 6380 & $Q$ & Alpha, Beta \\
\hline
\end{tabular}

* Performed by HEHF. 
IV. SURFACE WATER

\begin{tabular}{|c|c|c|c|}
\hline Location & EMA \# & Frequency & Analyses \\
\hline Gable Mounta in Pond & 1054 & Q & Alpha, Beta, Gamma scan, \\
\hline West Lake & 6133 & Q & Alpha, Beta, Garma scan, \\
\hline 8 Pond & 0015 & $Q$ & Alpha, Beta, Garma scan, \\
\hline FFTF Pond & 6467 & Q & Alpha, Beta, Gamma scan, \\
\hline
\end{tabular}


V. GROUND WATER

\begin{tabular}{|c|c|c|c|}
\hline \multirow[t]{2}{*}{ We 11 Number } & \multirow{2}{*}{$\frac{\text { EMA \# }}{600}$} & \multirow{2}{*}{ Frequency } & \multirow[t]{2}{*}{ Analyses $^{(a)}$} \\
\hline & & & \\
\hline$(699-) 50-7$ & $\star 4817$ & Q & \multirow{4}{*}{$\begin{array}{ll}{ }^{3} \mathrm{H}, \mathrm{NO}_{3}, \&, Y \\
3_{3} \mathrm{H}, \mathrm{NO}_{3}, B, Y \\
3_{3}, \mathrm{NO}_{3}, B, Y \\
3_{\mathrm{H},}, \mathrm{NO}_{3}\end{array}$} \\
\hline $50-8$ & $\star 4818$ & Q & \\
\hline$S 1-7 B$ & $\star 4819$ & $Q$ & \\
\hline \multirow[t]{2}{*}{$53-25$} & 4787 & $Q$ & \\
\hline & & A & WQ \\
\hline \multirow[t]{2}{*}{ S3-E12 } & 4553 & $Q$ & $3_{\mathrm{H}, \mathrm{NO}_{3}}$ \\
\hline & & A & WQ \\
\hline$S 6-E 4 \quad B$ & 4502 & $Q$ & ${ }^{3} \mathrm{H}, \mathrm{NO}_{3}, \mathrm{U}$ \\
\hline$S 6-E 4 \quad D$ & 4504 & $Q$ & ${ }^{3} \mathrm{H}, \mathrm{NO}_{3}, \mathrm{U}$ \\
\hline S6-E14 & 4580 & $Q$ & ${ }^{3} \mathrm{H}, \mathrm{NO}_{3}$ \\
\hline $57-34$ & 4427 & $Q$ & ${ }_{3}^{3}, \mathrm{NO}_{3}$ \\
\hline $58-19$ & 4421 & Q & ${ }^{3} \mathrm{H}, \mathrm{NO}_{3}$ \\
\hline$S 11-E 12 A \quad 0$ & 4552 & $Q$ & ${ }_{2}^{3} \mathrm{H}, \mathrm{NO}_{3}$ \\
\hline$S 11-E 12 A P$ & 4747 & $Q$ & ${ }_{3}^{3} \mathrm{H}, \mathrm{NO}_{3}$ \\
\hline$\$ 12-3$ & 4424 & $Q$ & ${ }^{3} \mathrm{H}, \mathrm{NO}_{3}$ \\
\hline$\$ 12-29$ & 4592 & $Q$ & $\mathrm{NO}_{3}$ \\
\hline$\$ 14-20 \mathrm{~A}$ & 4535 & $Q$ & $\mathrm{NO}_{3}$ \\
\hline$\$ 18-51$ & 4852 & $Q$ & $\mathrm{NO}_{3}, \mathrm{~F}$ \\
\hline$\$ 19-11$ & 4780 & SA & $\mathrm{NO}_{3}$ \\
\hline \multirow[t]{2}{*}{ S19-E13 } & 4802 & $Q$ & $\mathrm{NO}_{3}, \mathrm{~F}, \mathrm{U}$ \\
\hline & & A & WQ \\
\hline$\$ 24-19$ & 4510 & SA & $\mathrm{NO}_{3}$ \\
\hline S27-E14 & 4413 & M & $\mathrm{NO}_{3}, U, \mathrm{~F}, \mathrm{Cr}^{+6}$ \\
\hline$S 29-E 12$ & 4803 & $Q$ & $\mathrm{NO}_{3}, \mathrm{~F}, \mathrm{U}$ \\
\hline$S 30-E 15 \mathrm{~A}$ & 4804 & $Q$ & $\mathrm{NO}_{3}, \mathrm{~F}, \mathrm{U}, \mathrm{Cr}^{+6}$ \\
\hline \multirow[t]{2}{*}{$S 31-1 P$} & 4745 & $Q$ & ${ }^{3} \mathrm{H}, \mathrm{NO}_{3}$ \\
\hline & & A & WQ \\
\hline $1-18$ & 4513 & $Q$ & ${ }^{3} \mathrm{H}, \mathrm{NO}_{3}$ \\
\hline \multirow[t]{3}{*}{$2-3$} & $\star 4423$ & M & ${ }^{3} \mathrm{H}, \mathrm{NO}_{3}$ \\
\hline & & $Q$ & $B, \gamma$ \\
\hline & & A & WQ \\
\hline
\end{tabular}

(a) Water quality analyses (designated WQ) include the following: $\mathrm{pH}$, Conductance, $\mathrm{Ca}, \mathrm{Mg}, \mathrm{Na}, \mathrm{CO}_{3}, \mathrm{HCO}_{3}, \mathrm{~K}, \mathrm{~B}, \mathrm{NO}_{3}-\mathrm{N}, \mathrm{Cl}$, $\mathrm{SO}_{4}-\mathrm{S}$, and dissolved solids.

* FFTF Preoperational Survey. 
V. GROUND WATER (contd)

\begin{tabular}{|c|c|c|c|}
\hline We 11 Number & EMA \# & Frequency & Analyses $(a)$ \\
\hline$(699-) 2-7$ & 4758 & $A$ & WQ \\
\hline $2-33$ & 4526 & $Q$ & ${ }^{3} \mathrm{H}$, \\
\hline $3-45$ & 4593 & $Q$ & $\mathrm{NO}_{3}$ \\
\hline & & SA & \\
\hline $4-E 6$ & 4620 & $Q$ & \\
\hline $8-17$ & 4426 & $Q$ & , $\mathrm{NO}_{3}, \mathrm{r}$ \\
\hline $8-25$ & 4788 & $Q$ & I, $\mathrm{NO}_{3}, \mathrm{r}$ \\
\hline $8-32$ & 4420 & $Q$ & ${ }^{3} \mathrm{H}, \mathrm{NO}_{3}$ \\
\hline $9-E 2$ & 4519 & Q & $\mathrm{NO}_{3}$ \\
\hline $10-E 12$ & 4581 & $Q$ & ${ }^{3} \mathrm{H}, \mathrm{NO}_{3}$ \\
\hline $10-54$ & 4428 & $A$ & WQ \\
\hline $13-1 A$ & 4830 & Q & ${ }^{3} \mathrm{H}$, \\
\hline & & SA & Y \\
\hline $13-1 \quad B$ & 4831 & $Q$ & ${ }^{3} \mathrm{H}, \mathrm{NO}_{3}$ \\
\hline & & SA & $Y$ \\
\hline $13-1 C$ & 4854 & $Q$ & ${ }^{3} \mathrm{H}, \mathrm{NO}_{3}$ \\
\hline & & SA & $Y$ \\
\hline $13-64$ & 4429 & Q & ${ }^{3} \mathrm{H}, \quad \mathrm{NO}_{3}$ \\
\hline $14-E 6 P$ & 4700 & $Q$ & \\
\hline $14-E 6 \quad 0$ & 4701 & $Q$ & \\
\hline $14-E 6 R$ & 4702 & $Q$ & \\
\hline $14-E 6 S$ & 4703 & $Q$ & ${ }^{3} \mathrm{H}, \mathrm{NO}_{3}$ \\
\hline 14-E6 T & 4766 & $Q$ & \\
\hline $14-38$ & 4527 & $Q$ & \\
\hline $14-47$ & 4608 & $Q$ & ${ }^{3} \mathrm{H}, \mathrm{NO}_{3}$ \\
\hline & & $A$ & WQ \\
\hline $15-158$ & 4810 & $Q$ & $8,{ }^{3} \mathrm{H}, \mathrm{NO}_{3}, Y$ \\
\hline $15-26$ & 4464 & $Q$ & $B,{ }^{3} H, N O_{3}, Y$ \\
\hline & & $A$ & WQ \\
\hline $17-5$ & 4422 & $Q$ & $B,{ }^{3} \mathrm{H}, \mathrm{NO}_{3}, \mathrm{r}$ \\
\hline $17-70$ & 4531 & $Q$ & ${ }^{3} \mathrm{H}, \mathrm{NO}_{3}$ \\
\hline $19-43$ & 4417 & $Q$ & ${ }^{3} \mathrm{H}, \mathrm{NO}_{3}$ \\
\hline & & $A$ & WQ \\
\hline
\end{tabular}

(a) Water quality analyses (designated WQ) include the following: $\mathrm{pH}$, Conductance, $\mathrm{Ca}, \mathrm{Mg}, \mathrm{Na}, \mathrm{CO}_{3}, \mathrm{HCO}_{3}, \mathrm{~K}, \mathrm{~B}, \mathrm{NO}_{3}-\mathrm{N}, \mathrm{Cl}, \mathrm{SO}_{4}-\mathrm{S}$, and dissolved solids. 
V. GROUND WATER (contd)

\begin{tabular}{|c|c|c|c|}
\hline Well Number & EMA \# & Frequency & Analyses ${ }^{(a)}$ \\
\hline$(699-) 19-47$ A & 4609 & $Q$ & ${ }^{3} \mathrm{H}, \mathrm{NO}_{3}$ \\
\hline $19-58$ & 4528 & $Q$ & $\mathrm{NO}_{3}$ \\
\hline \multirow[t]{2}{*}{$19-88$} & 4522 & $Q$ & $\mathrm{NO}_{3}$ \\
\hline & & A & $F$ \\
\hline \multirow[t]{2}{*}{ 20-E5 A } & 4838 & $Q$ & ${ }^{3} \mathrm{H}, \mathrm{NO}_{3}, Y$ \\
\hline & & A & WQ \\
\hline 20-E5P & 4705 & $Q$ & ${ }^{3} \mathrm{H}, \mathrm{NO}_{3}$ \\
\hline $20-E 5 \quad Q$ & 4706 & 0 & ${ }^{3} \mathrm{H}, \mathrm{NO}_{3}$ \\
\hline 20-E5 R & 4707 & $Q$ & ${ }^{3} \mathrm{H}, \mathrm{NO}_{3}$ \\
\hline $20-E 120$ & 4567 & $Q$ & ${ }^{3} \mathrm{H}, \mathrm{NO}_{3}$ \\
\hline 20-E12P & 4611 & $Q$ & \\
\hline $20-20$ & 4418 & $Q$ & $\mathrm{~B},{ }^{3} \mathrm{H}, \mathrm{NO}_{3}$ \\
\hline \multirow[t]{2}{*}{$20-39$} & 4559 & $Q$ & ${ }^{3} \mathrm{H}, \mathrm{NO}_{3}$ \\
\hline & & A & $\gamma$ \\
\hline \multirow[t]{2}{*}{$22-70$} & 4595 & $Q$ & ${ }^{3} \mathrm{H}, \mathrm{NO}_{3}$ \\
\hline & & A & Y \\
\hline $20-82$ & 4529 & $Q$ & ${ }^{3} \mathrm{H}, \mathrm{NO}_{3}$ \\
\hline $23-7$ & 4855 & M & ${ }^{3} \mathrm{H}, \mathrm{NO}_{3}$ \\
\hline $24-1 P$ & 4710 & $Q$ & ${ }_{2}^{3} \mathrm{H}, \mathrm{NO}_{3}$ \\
\hline $24-1 Q$ & 4711 & $Q$ & ${ }_{2}^{3} \mathrm{H}, \mathrm{NO}_{3}$ \\
\hline $24-1 R$ & 4712 & $Q$ & ${ }^{3} \mathrm{H}, \mathrm{NO}_{3}$ \\
\hline $24-1 S$ & 4713 & $Q$ & ${ }^{3} \mathrm{H}, \mathrm{NO}_{3}$ \\
\hline $24-1 T$ & 4764 & $Q$ & ${ }^{3} \mathrm{H}, \mathrm{NO}_{3}$ \\
\hline $24-33$ & 4416 & $Q$ & $\mathrm{~B},{ }^{3} \mathrm{H}, \mathrm{NO}_{3}$, \\
\hline $24-46$ & 4525 & $Q$ & ${ }^{3} \mathrm{H}, \mathrm{NO}_{3}$ \\
\hline $25-55$ & 4415 & $Q$ & ${ }^{3} \mathrm{H}, \mathrm{NO}_{3}$ \\
\hline \multirow[t]{2}{*}{$25-70$} & 4452 & SA & ${ }^{3} \mathrm{H}, \mathrm{NO}_{3}$ \\
\hline & & A & WQ \\
\hline \multirow[t]{2}{*}{$26-15$} & 4518 & $Q$ & $\mathrm{~B},{ }^{3} \mathrm{H}, \mathrm{NO}_{3}$, \\
\hline & & A & WQ \\
\hline \multirow[t]{2}{*}{$26-89$} & 4598 & SA & $\mathrm{NO}_{3}$ \\
\hline & & A & WQ \\
\hline $27-4$ & 4857 & M & ${ }^{3} \mathrm{H}, \mathrm{NO}_{3}$ \\
\hline
\end{tabular}

(a) Water quality analyses (designated WQ) include the following: $\mathrm{pH}$, Conductance, $\mathrm{Ca}, \mathrm{Mg}, \mathrm{Na}, \mathrm{CO}_{3}, \mathrm{HCO}_{3}, \mathrm{~K}, \mathrm{~B}, \mathrm{NO}_{3}-\mathrm{N}, \mathrm{C}, \mathrm{SO}_{4}-\mathrm{S}$, and dissolved solids. 
V. GROUND WATER (contd)

\begin{tabular}{|c|c|c|c|}
\hline Well Number & EMA \# & Frequency & Analyses ${ }^{(a)}$ \\
\hline$(699-) 27-8$ & 4557 & Q & $\mathrm{B},{ }^{3} \mathrm{H}, \mathrm{NO}_{3}, \mathrm{\gamma}$ \\
\hline $29-78$ & 4594 & $Q$ & ${ }^{3} \mathrm{H}, \mathrm{NO}_{3}$ \\
\hline $28-400$ & 4481 & $Q$ & ${ }^{3} \mathrm{H}, \mathrm{NO}_{3}, \mathrm{Y}$ \\
\hline $28-40 P$ & 4754 & $Q$ & ${ }^{3} \mathrm{H}, \mathrm{NO}_{3}^{3}, \mathrm{Y}$ \\
\hline $28-52$ & 4521 & $Q$ & ${ }^{3} \mathrm{H}, \mathrm{NO}_{3}, \mathrm{Y}$ \\
\hline $31-310$ & 4471 & $Q$ & ${ }^{3} \mathrm{H}, \mathrm{NO}_{3}, \mathrm{Y}$ \\
\hline $31-31 P$ & 4738 & $Q$ & ${ }^{3} \mathrm{H}, \mathrm{NO}_{3}, Y$ \\
\hline $31-53 \quad B$ & 4520 & $S A$ & ${ }^{3} \mathrm{H}, \mathrm{NO}_{3}, Y$ \\
\hline & & A & WQ \\
\hline $31-650$ & 4495 & $Q$ & \\
\hline $32-22$ & 4794 & $Q$ & $\mathrm{H}, \mathrm{NO}_{3}, \gamma$ \\
\hline $32-42$ & 4777 & $Q$ & \\
\hline $32-43$ & 4778 & $Q$ & $\mathrm{NO}_{3}, Y$ \\
\hline $32-62$ & 4550 & $Q$ & $10_{3}^{2}, \alpha$ \\
\hline $32-70$ & 4492 & $Q$ & $\mathrm{~B},{ }^{3} \mathrm{H}, \mathrm{NO}_{3}, \gamma$ \\
\hline $32-72$ & 4491 & $Q$ & $\mathrm{NO}_{3}, \bar{Y}$ \\
\hline $32-77$ & 4446 & $Q$ & $\mathrm{H}, \mathrm{NO}_{3}, \mathrm{Y}$ \\
\hline $33-42$ & 4779 & $Q$ & $\mathrm{~B},{ }^{3} \mathrm{H}, \mathrm{NO}_{3}, \gamma$ \\
\hline $33-56$ & 4523 & $Q$ & $\mathrm{~B}, \alpha,{ }^{3} \mathrm{H}, \mathrm{NO}_{3}$, \\
\hline $34-39 A$ & 4448 & $Q$ & $\mathrm{~B},{ }^{3} \mathrm{H}, \mathrm{NO}_{3}, \mathrm{Y}$ \\
\hline $34-41$ & 4789 & $Q$ & $\mathrm{O}_{3}, \dot{\gamma}$ \\
\hline $34-42$ & 4790 & $Q$ & $\mathrm{~B},{ }^{3} \mathrm{H}, \mathrm{NO}_{3}, \gamma$ \\
\hline & & A & WQ \\
\hline $34-51$ & 4414 & $Q$ & $\mathrm{~B},{ }^{3} \mathrm{H}, \mathrm{NO}_{3}, \mathrm{Y}$ \\
\hline $34-88$ & 4439 & $Q$ & ${ }^{3} \mathrm{H}, \mathrm{NO}_{3}$ \\
\hline $35-9$ & 4419 & $Q$ & $\mathrm{~B},{ }^{3} \mathrm{H}, \mathrm{NO}_{3}, \gamma$ \\
\hline $35-66$ & 4494 & $Q$ & $\mathrm{~B},{ }^{3} \mathrm{H}, \mathrm{NO}_{3}, Y$ \\
\hline $35-70$ & 4441 & $Q$ & $\mathrm{~B},{ }^{3} \mathrm{H}, \mathrm{NO}_{3}, \gamma$ \\
\hline & & A & WQ \\
\hline $35-78$ & 4445 & $Q$ & $\mathrm{~B},{ }^{3} \mathrm{H}, \mathrm{NO}_{3}, Y$ \\
\hline $36-46 P$ & 4751 & $Q$ & ${ }^{3} \mathrm{H}, \mathrm{NO}_{3}$ \\
\hline & & SA & Y \\
\hline $36-46 Q$ & 4752 & $Q$ & ${ }^{3} \mathrm{H}, \mathrm{NO}_{3}$ \\
\hline & & $S A$ & $\gamma$ \\
\hline $36-61 A$ & 4447 & $Q$ & $\mathrm{NO}_{3}$ \\
\hline
\end{tabular}

(a) Water quality analyses (designated WQ) include the following: $\mathrm{pH}$, Conductance, $\mathrm{Ca}, \mathrm{Mg}, \mathrm{Na}, \mathrm{CO}_{3}, \mathrm{HCO}_{3}, \mathrm{~K}, \mathrm{~B}, \mathrm{NO}_{3}-\mathrm{N}, \mathrm{Cl}$, $\mathrm{SO}_{4}-\mathrm{S}$, and dissolved solids. 
V. GROUND WATER (contd)

\begin{tabular}{|c|c|c|c|}
\hline We 11 Number & EMA \# & Frequency & Analyses $^{(a)}$ \\
\hline$(699-) 36-61$ B & 4549 & $Q$ & $3_{\mathrm{H}, \mathrm{NO}}$ \\
\hline \multirow[t]{2}{*}{$36-93$} & 4579 & $S A$ & ${ }^{3} \mathrm{H}, \mathrm{NO}_{3}$ \\
\hline & & $A$ & WQ \\
\hline $37-43$ & 4480 & $Q$ & $\mathrm{~B},{ }^{3} \mathrm{H}, \mathrm{NO}_{3}, \mathrm{Y}$ \\
\hline $37-82 A$ & 4554 & $Q$ & ${ }^{3} \mathrm{H}, \mathrm{NO}_{3}$ \\
\hline \multirow[t]{2}{*}{$38-65$} & 4546 & $Q$ & ${ }^{3} \mathrm{H}, \mathrm{NO}_{3}$ \\
\hline & & $S A$ & $\gamma$ \\
\hline $38-70$ & 4493 & $Q$ & $\mathrm{~B},{ }^{3} \mathrm{H}, \mathrm{NO}_{3}, \mathrm{Y}$ \\
\hline $39-39$ & 4791 & $S A$ & $\mathrm{~B},{ }^{3} \mathrm{H}, \mathrm{NO}_{3}, \mathrm{Y}$ \\
\hline $39-79$ & 4444 & $Q$ & $\mathrm{~B},{ }^{3} \mathrm{H}, \mathrm{NO}_{3}, \gamma$ \\
\hline \multirow[t]{2}{*}{$40-1$} & 4566 & Q & ${ }^{3} \mathrm{H}, \mathrm{NO}_{3}$ \\
\hline & & A & $Y$, WQ \\
\hline \multirow[t]{2}{*}{$40-33$} & 4431 & $Q$ & ${ }^{3} \mathrm{H}, \mathrm{NO}_{3}$ \\
\hline & & A & $Y$, WQ \\
\hline $40-62$ & 4458 & $Q$ & ${ }^{3} \mathrm{H}, \mathrm{NO}_{3}$ \\
\hline $41-1$ & 4858 & M & \\
\hline $41-23$ & 4430 & $Q$ & $\mathrm{~B},{ }^{3} \mathrm{H}, \mathrm{NO}_{3}, \mathrm{Y}$ \\
\hline $42-2$ & 4859 & M & \\
\hline $42-12$ & 4517 & $Q$ & $\mathrm{~B},{ }^{3} \mathrm{H}, \mathrm{NO}_{3}, \gamma$ \\
\hline $43-3$ & 4861 & M & ${ }^{3} \mathrm{H}, \mathrm{NO}_{3}$ \\
\hline $43-88$ & 4836 & $Q$ & ${ }^{3} \mathrm{H}, \mathrm{NO}_{3}$ \\
\hline $44-64$ & 4548 & $Q$ & ${ }^{3} \mathrm{H}, \mathrm{NO}_{3},{ }^{\gamma}$ \\
\hline $45-4$ & 4862 & M & ${ }^{3} \mathrm{H}, \mathrm{NO}_{3}$ \\
\hline $45-42$ & 4450 & $Q$ & $\mathrm{~B},{ }^{3} \mathrm{H}, \mathrm{NO}_{3}, Y$ \\
\hline & & A & \\
\hline $45-69$ & 4449 & $Q$ & ${ }^{3} \mathrm{H}, \mathrm{NO}_{3}$ \\
\hline & & SA & $8, \gamma$ \\
\hline & & A & \\
\hline $46-5$ & 4863 & M & ${ }^{3} \mathrm{H}, \mathrm{NO}_{3}$ \\
\hline $46-21$ & 4479 & $Q$ & ${ }_{3}^{3}, \mathrm{NO}_{3}$ \\
\hline $47-6$ & 4864 & M & \\
\hline $47-35$ & 4478 & $Q$ & ${ }^{3} \mathrm{H}, \mathrm{NO}_{3}$ \\
\hline
\end{tabular}

(a) Water quality analyses (designated WQ) include the following:

$\mathrm{pH}$, Conductance, $\mathrm{Ca}, \mathrm{Mg}, \mathrm{Na}, \mathrm{CO}_{3}, \mathrm{HCO}_{3}, \mathrm{~K}, \mathrm{~B}, \mathrm{NO}_{3}-\mathrm{N}, \mathrm{Cl}$,

$\mathrm{SO}_{4}-\mathrm{S}$, and dissolved solids. 
V. GROUND WATER (contd)

\begin{tabular}{|c|c|c|c|}
\hline We 11 Number & EMA \# & Frequency & Analyses ${ }^{(a)}$ \\
\hline \multirow[t]{2}{*}{$(699-) 47-46$} & \multirow[t]{2}{*}{4564} & $Q$ & ${ }^{3} \mathrm{H}, \mathrm{NO}_{3}$ \\
\hline & & $S A$ & $\beta, \gamma$ \\
\hline $47-60$ & 4434 & $Q$ & $\mathrm{~B},{ }^{3} \mathrm{H}, \mathrm{NO}_{3}, \gamma$ \\
\hline $48-7$ & 4756 & $Q$ & ${ }^{3} \mathrm{H}, \mathrm{NO}_{3}$ \\
\hline $48-18$ & 4830 & $Q$ & ${ }^{3} \mathrm{H}, \mathrm{NO}_{3}$ \\
\hline $48-71$ & 4487 & $Q$ & ${ }^{3} \mathrm{H}, \mathrm{NO}_{3}$ \\
\hline \multirow[t]{2}{*}{$49-13$} & \multirow[t]{2}{*}{4771} & $Q$ & ${ }^{3} \mathrm{H}, \mathrm{NO}_{3}$ \\
\hline & & A & WQ \\
\hline $49-28$ & 4816 & $Q$ & \\
\hline $49-55$ & 4562 & $Q$ & $\mathrm{~B},{ }^{3} \mathrm{H}, \mathrm{NO}_{3}$ \\
\hline $49-57$ & 4485 & $Q$ & $\mathrm{~B},{ }^{3} \mathrm{H}, \mathrm{NO}_{3}, \gamma$ \\
\hline \multirow[t]{2}{*}{$49-79$} & \multirow[t]{2}{*}{4443} & $Q$ & ${ }^{3} \mathrm{H}, \quad \mathrm{NO}_{3}$ \\
\hline & & A & WQ \\
\hline $50-19$ & 4776 & $S A$ & $\mathrm{NO}_{3}$ \\
\hline \multirow[t]{2}{*}{$50-28 B$} & \multirow[t]{2}{*}{4844} & $Q$ & ${ }^{3} \mathrm{H}, \mathrm{NO}_{3}$ \\
\hline & & A & WQ \\
\hline $50-30$ & 4451 & $Q$ & ${ }^{3} \mathrm{H}, \mathrm{NO}_{3}$ \\
\hline $50-42$ & 4460 & $Q$ & \\
\hline \multirow[t]{2}{*}{$50-53$} & \multirow[t]{2}{*}{4473} & $Q$ & $\mathrm{~B},{ }^{3} \mathrm{H}, \mathrm{NO}_{3}, \gamma$ \\
\hline & & $A$ & WQ \\
\hline $50-85$ & 4497 & $Q$ & ${ }^{3} \mathrm{H}, \mathrm{NO}_{3}$ \\
\hline $51-63$ & 4488 & $Q$ & ${ }^{3} \mathrm{H}, \mathrm{NO}_{3}$ \\
\hline $51-75$ & 4496 & $Q$ & ${ }^{3} \mathrm{H}, \mathrm{NO}_{3}$ \\
\hline \multirow[t]{2}{*}{$53-35$} & \multirow[t]{2}{*}{4637} & $Q$ & ${ }^{3} \mathrm{H}, \mathrm{NO}_{3}$ \\
\hline & & $A$ & $\gamma$ \\
\hline $53-47$ & 4774 & $Q$ & ${ }^{3} \mathrm{H}, \mathrm{NO}_{3}$ \\
\hline $53-55 A$ & 4563 & $Q$ & ${ }^{3} \mathrm{H}, \mathrm{NO}_{3}$ \\
\hline $53-103$ & 4772 & $Q$ & ${ }^{3} \mathrm{H}, \mathrm{NO}_{3}$ \\
\hline $54-34$ & 4638 & $Q$ & ${ }^{3} \mathrm{H}, \mathrm{NO}_{3}$ \\
\hline $54-37 \mathrm{~A}$ & 4853 & $Q$ & ${ }^{3} \mathrm{H}, \mathrm{NO}_{3}$ \\
\hline \multirow[t]{2}{*}{$54-42$} & \multirow[t]{2}{*}{4432} & $Q$ & $\mathrm{NO}_{3}$ \\
\hline & & SA & \\
\hline $54-45$ & 4811 & $Q$ & ${ }^{3} \mathrm{H}, \mathrm{NO}_{3}$ \\
\hline
\end{tabular}

(a) Water quality analyses (designated $W Q$ ) include the following: $\mathrm{pH}$, Conductance, $\mathrm{Ca}, \mathrm{Mg}, \mathrm{Na}, \mathrm{CO}_{3}, \mathrm{HCO}_{3}, \mathrm{~K}, \mathrm{~B}, \mathrm{NO}_{3}-\mathrm{N}, \mathrm{Cl}$, $\mathrm{SO}_{4}-\mathrm{S}$, and dissolved solids. 
V. GROUND WATER (contd)

\begin{tabular}{|c|c|c|c|}
\hline We 11 Number & EMA \# & Frequency & Analyses \\
\hline$(699-) 54-57$ & 4469 & $Q$ & ${ }^{3} \mathrm{H}, \mathrm{NO}_{3}$ \\
\hline $55-40$ & 4639 & $Q$ & ${ }^{3} \mathrm{H}, \mathrm{NO}_{3}$ \\
\hline $55-44$ & 4640 & 0 & ${ }_{3}^{3} \mathrm{H}, \mathrm{NO}_{3}$ \\
\hline $55-50$ AO & 4433 & $Q$ & ${ }^{3} \mathrm{H}, \mathrm{NO}_{3}$ \\
\hline \multirow[t]{3}{*}{$55-50 \mathrm{C}$} & \multirow[t]{3}{*}{4483} & $Q$ & ${ }^{3} \mathrm{H}, \mathrm{NO}_{3}$ \\
\hline & & SA & $Y$ \\
\hline & & A & WQ \\
\hline $55-50 \quad D$ & 4484 & $Q$ & ${ }_{3}^{3} \mathrm{H}, \mathrm{NO}_{3}$ \\
\hline $55-70$ & 4442 & $Q$ & ${ }^{3} \mathrm{H}, \mathrm{NO}_{3}$ \\
\hline $55-76$ & 4533 & $Q$ & $\mathrm{NO}_{3}$ \\
\hline $55-89$ & 4453 & $Q$ & $\mathrm{NO}_{3}$ \\
\hline $56-43$ & 4650 & $Q$ & ${ }^{3} \mathrm{H}, \mathrm{NO}_{3}$ \\
\hline $57-25 A$ & 4856 & $Q$ & ${ }_{2}^{3} \mathrm{H}$, \\
\hline $57-29 A$ & 4462 & $Q$ & ${ }^{3} \mathrm{H}, \mathrm{NO}_{3}$ \\
\hline $57-83$ & 4558 & $Q$ & $\mathrm{NO}_{3}$ \\
\hline $58-24$ & 4652 & $Q$ & $0_{3}$ \\
\hline $59-32$ & 4815 & $Q$ & $\mathrm{O}_{3}$ \\
\hline \multirow[t]{2}{*}{$59-58$} & \multirow[t]{2}{*}{4827} & $Q$ & ${ }^{3} \mathrm{H}, \mathrm{NO}_{3}$ \\
\hline & & A & WQ \\
\hline $59-80 \quad$ B & 4437 & $Q$ & $\mathrm{NO}_{3}$ \\
\hline $60-32$ & 4814 & $Q$ & ${ }^{3} \mathrm{H}, \mathrm{NO}_{3}$ \\
\hline $60-57$ & 4826 & $Q$ & ${ }_{3}^{3} \mathrm{H}, \mathrm{NO}_{3}$ \\
\hline $60-60$ & 4435 & $Q$ & ${ }^{3} \mathrm{H}, \mathrm{NO}_{3}$ \\
\hline $61-37$ & 4694 & $Q$ & $\mathrm{NO}_{3}$ \\
\hline $61-41$ & 4653 & $Q$ & ${ }_{3}^{3} \mathrm{H}, \mathrm{NO}_{3}$ \\
\hline $61-62$ & 4825 & $Q$ & ${ }_{3}^{\mathrm{H}}, \mathrm{NO}_{3}$ \\
\hline $61-66$ & 4474 & $Q$ & ${ }^{3} \mathrm{H}, \mathrm{NO}_{3}$ \\
\hline $62-31$ & 4813 & $Q$ & $\mathrm{NO}_{3}$ \\
\hline $62-43 \quad F$ & 4537 & $Q$ & \\
\hline $63-25$ & 4499 & $Q$ & ${ }_{2}^{3} \mathrm{H}, \mathrm{NO}_{3}$ \\
\hline $63-51$ & 4845 & $S A$ & ${ }^{3} \mathrm{H}, \mathrm{NO}_{3}$ \\
\hline $63-55$ & 4823 & $Q$ & \\
\hline $63-58$ & 4822 & $Q$ & \\
\hline $63-90$ & 4436 & $Q$ & ${ }^{3} \mathrm{H}, \mathrm{NO}_{3}$ \\
\hline
\end{tabular}

(a) Water quality analyses (designated WQ) include the following: $\mathrm{pH}$, Conductance, $\mathrm{Ca}, \mathrm{Mg}, \mathrm{Na}, \mathrm{CO}_{3}, \mathrm{HCO}_{3}, \mathrm{~K}, \mathrm{~B}, \mathrm{NO}_{3}-\mathrm{N}, \mathrm{Cl}$, $\mathrm{SO}_{4}-\mathrm{S}$, and dissolved solids. 
V. GROUND WATER (contd)

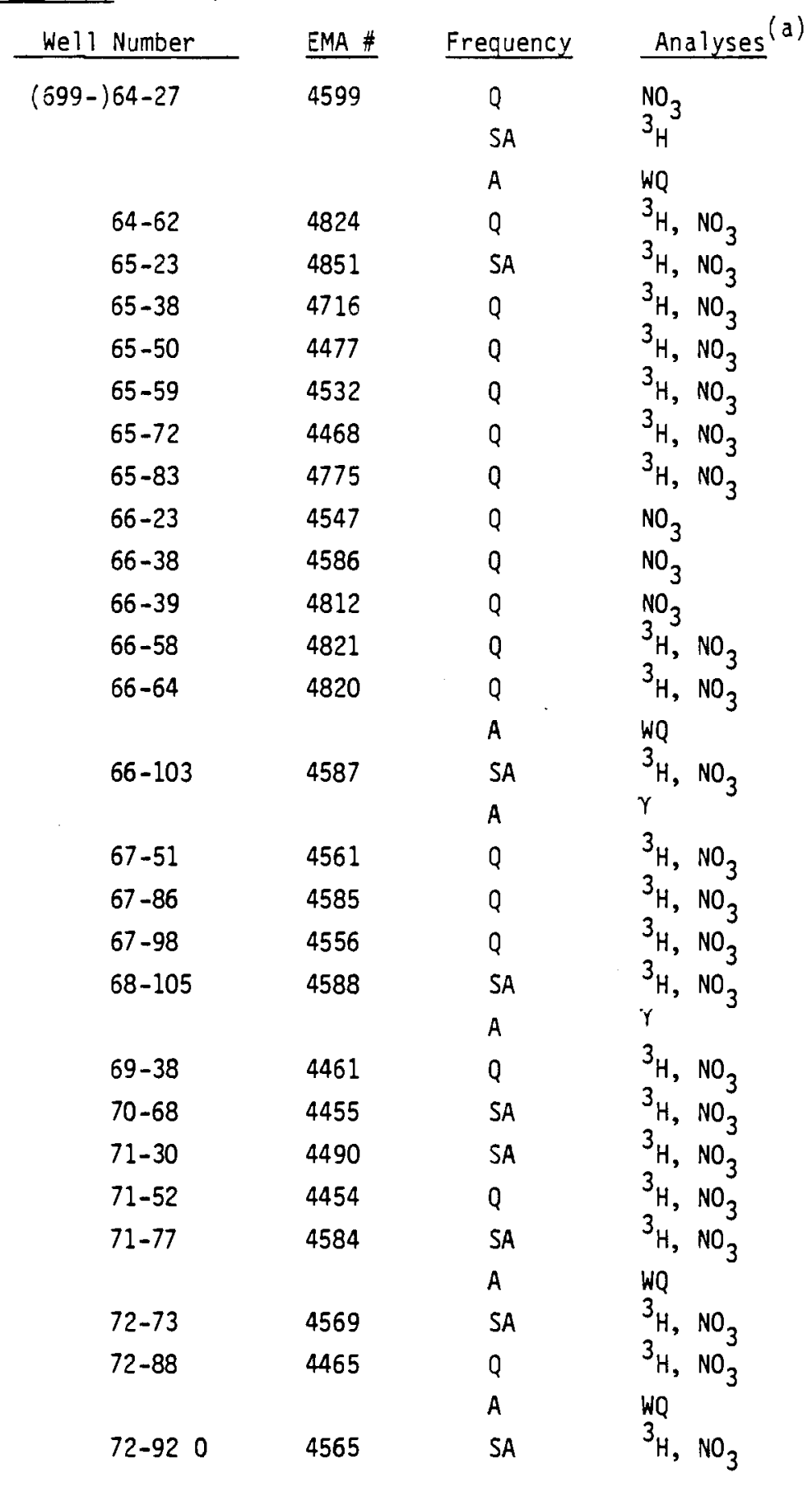

(a) Water quality analyses (designated WQ) include the following: $\mathrm{pH}$, Conductance, $\mathrm{Ca}, \mathrm{Mg}, \mathrm{Na}, \mathrm{CO}_{3}, \mathrm{HCO}_{3}, \mathrm{~K}, \mathrm{~B}, \mathrm{NO}_{3}-\mathrm{N}, \mathrm{Cl}, \mathrm{SO}_{4}-\mathrm{S}$, and dissolved solids. 
V. GROUND WATER (contd)

\begin{tabular}{|c|c|c|c|}
\hline We 11 Number & EMA \# & Frequency & Analyses $(a)$ \\
\hline$(699-) 72-98$ & 4463 & SA & ${ }^{3} \mathrm{H}, \mathrm{NO}_{3}$ \\
\hline $73-61$ & 4583 & $Q$ & ${ }^{3} \mathrm{H}, \mathrm{NO}_{3}$ \\
\hline \multirow[t]{2}{*}{$74-44$} & 4516 & $Q$ & ${ }^{3} \mathrm{H}, \mathrm{NO}_{3}^{2}$ \\
\hline & & A & WQ \\
\hline $74-60$ & 4583 & SA & $\mathrm{NO}_{3}$ \\
\hline $77-36$ & 4500 & $Q$ & ${ }^{3} \mathrm{H}, \mathrm{NO}_{3}$ \\
\hline $77-54$ & 4512 & $Q$ & $\mathrm{NO}_{3}$ \\
\hline \multirow[t]{2}{*}{$78-62$} & 4511 & SA & $\mathrm{NO}_{3}$ \\
\hline & & $A$ & WQ \\
\hline $80-43 P$ & 4760 & SA & $\mathrm{NO}_{3}$ \\
\hline $80-43 Q$ & 4761 & SA & $\mathrm{NO}_{3}$ \\
\hline $80-43 R$ & 4762 & SA & $\mathrm{NO}_{3}$ \\
\hline $80-43 \mathrm{~S}$ & 4763 & SA & $\mathrm{NO}_{3}$ \\
\hline \multirow[t]{2}{*}{$81-58$} & 4597 & $Q$ & ${ }^{3} \mathrm{H}, \mathrm{NO}_{3}$ \\
\hline & & $A$ & WQ \\
\hline $83-47$ & 4515 & SA & \\
\hline $84-35 A 0$ & 4596 & SA & $\mathrm{H}, \mathrm{NO}_{3}$ \\
\hline \multirow[t]{2}{*}{$87-55$} & 4792 & $Q$ & $\mathrm{H}, \mathrm{NO}_{3}$ \\
\hline & & $A$ & WQ \\
\hline \multirow[t]{2}{*}{$89-35$} & 4571 & $Q$ & $\mathrm{NO}_{3}$ \\
\hline & & $A$ & WQ \\
\hline \multirow[t]{2}{*}{$90-45$} & 4770 & $Q$ & H \\
\hline & & SA & $\mathrm{NO}_{3}$ \\
\hline $96-49$ & 4591 & $Q$ & ${ }^{3} \mathrm{H}, \mathrm{NO}_{3}$ \\
\hline $97-43$ & 4590 & Q & \\
\hline $97-51 \mathrm{~A}$ & 4728 & $Q$ & \\
\hline $101-48 \quad B$ & 4846 & SA & ${ }^{3} \mathrm{H}, \mathrm{NO}_{3}$ \\
\hline \multicolumn{4}{|c|}{100 Area We11s } \\
\hline$(199-) B 3-1$ & 1851 & SA & ${ }^{3} \mathrm{H}$ \\
\hline$B 3-2 P$ & 1856 & SA & ${ }^{3} \mathrm{H}, \mathrm{NO}_{3}$ \\
\hline$B 3-2 Q$ & 1857 & SA & \\
\hline $84-1$ & 1853 & $Q$ & ${ }^{3} \mathrm{H}, \mathrm{NO}_{3}$ \\
\hline$B 4-2$ & 1854 & $Q$ & ${ }^{3} \mathrm{H}, \mathrm{NO}_{3}$ \\
\hline
\end{tabular}

(a) Water quality analyses (designated WQ) include the following: $\mathrm{pH}$, Conductance, $\mathrm{Ca}, \mathrm{Mg}, \mathrm{Na}, \mathrm{CO}_{3}, \mathrm{HCO}_{3}, \mathrm{~K}, \mathrm{~B}, \mathrm{NO}_{3}-\mathrm{N}, \mathrm{Cl}, \mathrm{SO}_{4}-\mathrm{S}$, and dissolved solids. 
V. GROUND WATER (contd)

\begin{tabular}{|c|c|c|c|}
\hline We11 Number & EMA \# & Frequency & Analyses (a) \\
\hline$(199-) B 4-3$ & 1855 & Q & $3_{\mathrm{H}, \mathrm{NO}}$ \\
\hline$B 4-4$ & 1891 & SA & $\mathrm{B},{ }^{3} \mathrm{H}, \mathrm{NO}_{3}, \mathrm{Y}$ \\
\hline$B 5-1$ & 1895 & $Q$ & ${ }^{3} \mathrm{H}, \mathrm{NO}_{3}$ \\
\hline B9-1 & 1893 & $S A^{\prime}$ & ${ }^{3} \mathrm{H}, \mathrm{NO}_{3}$ \\
\hline $02-5$ & 1894 & $Q$ & ${ }^{3} \mathrm{H}, \mathrm{NO}_{3}$ \\
\hline $05-12$ & 1892 & Q & $\mathrm{B},{ }^{3} \mathrm{H}, \mathrm{NO}_{3}, \mathrm{Y}$ \\
\hline $08-3$ & 1862 & $Q$ & ${ }^{3} \mathrm{H}, \mathrm{NO}_{3}$ \\
\hline$F 5-1$ & 1865 & $Q$ & ${ }^{3} \mathrm{H}, \mathrm{NO}_{3}$ \\
\hline$F 5-3$ & 1867 & $Q$ & ${ }^{3} \mathrm{H}, \mathrm{NO}_{3}$ \\
\hline F5-4 & 1868 & $Q$ & ${ }_{3}^{3} \mathrm{H}, \mathrm{NO}_{3}$ \\
\hline F5-6 & 1870 & $Q$ & ${ }^{3} \mathrm{H}, \mathrm{NO}_{3}$ \\
\hline F7-1 & 1871 & $Q$ & ${ }_{3}^{3} \mathrm{H}, \mathrm{NO}_{3}$ \\
\hline F8-1 & 1888 & $Q$ & ${ }_{3}^{3} \mathrm{H}, \mathrm{NO}_{3}$ \\
\hline$F 8-2$ & 1889 & $Q$ & ${ }^{3} \mathrm{H}, \mathrm{NO}_{3}$ \\
\hline H3-1 & 1890 & $Q$ & ${ }^{3} \mathrm{H}, \mathrm{NO}_{3}$ \\
\hline $\mathrm{H} 4-3$ & 1877 & M & $\begin{array}{l}\mathrm{B}, \mathrm{U},{ }^{3} \mathrm{H}, \mathrm{NO}_{3}, \mathrm{Cr}^{+6} \\
\mathrm{~F}, \mathrm{Cu}\end{array}$ \\
\hline & & $Q$ & $\gamma$ \\
\hline & & A & WQ \\
\hline$k-11$ & 1882 & Q & ${ }^{3} \mathrm{H}, \mathrm{NO}_{3}$ \\
\hline & & $S A$ & $\gamma$ \\
\hline$k-19$ & 1884 & $Q$ & ${ }^{3} \mathrm{H}, \mathrm{NO}_{3}$ \\
\hline$k-20$ & 1885 & $Q$ & ${ }^{3} \mathrm{H}, \mathrm{NO}_{3}$ \\
\hline$k-22$ & 1887 & $Q$ & ${ }^{3} \mathrm{H}, \mathrm{NO}_{3}$ \\
\hline$N-1$ & 1908 & $Q$ & ${ }^{3} \mathrm{H}, \mathrm{NO}_{3}, \mathrm{Y}$ \\
\hline & & SA & Sr \\
\hline$N-2$ & 1904 & $Q$ & ${ }^{3} \mathrm{H}, \mathrm{NO}_{3}, \mathrm{Y}$ \\
\hline & & SA & Sr \\
\hline $\mathrm{N}-30$ & 1896 & $Q$ & $B,{ }^{3} \mathrm{H}, \gamma$ \\
\hline & & SA & $S r$ \\
\hline$N-4$ & 1899 & $Q$ & $\mathrm{~B},{ }^{3} \mathrm{H}, \mathrm{NO}_{3}, \gamma$ \\
\hline & & SA & \\
\hline$N-5$ & 1909 & $Q$ & ${ }^{3} \mathrm{H}, \mathrm{NO}_{3}, \mathrm{Y}$ \\
\hline & & SA & $\mathrm{Sr}$ \\
\hline
\end{tabular}

(a) Water quality analyses (designated WQ) include the following: $\mathrm{PH}$, Conductance, $\mathrm{Ca}, \mathrm{Mg}, \mathrm{Na}, \mathrm{CO}_{3}, \mathrm{HCO}_{3}, \mathrm{~K}, \mathrm{~B}, \mathrm{NO}_{3}-\mathrm{N}, \mathrm{Cl}$, $\mathrm{SO}_{4}-\mathrm{S}$, and dissolved solids. 
V. GROUND WATER (contd)

\begin{tabular}{|c|c|c|c|}
\hline We11 Number & EMA \# & Frequency & Analyses (a) \\
\hline \multirow[t]{2}{*}{$(199-) N-6$} & 1901 & $Q$ & $B,{ }^{3} \mathrm{H}, \gamma$ \\
\hline & & $S A$ & $S r$ \\
\hline \multirow[t]{2}{*}{$N-7$} & 1910 & $Q$ & ${ }^{3} \mathrm{H}, \mathrm{NO}_{3}, \gamma$ \\
\hline & & SA & $S r$ \\
\hline \multirow[t]{2}{*}{$N-10 P$} & 1900 & $Q$ & $\mathrm{~B},{ }^{3} \mathrm{H}, \mathrm{NO}_{3}, \gamma$ \\
\hline & & SA & $\mathrm{Sr}$. \\
\hline \multirow[t]{2}{*}{$\mathrm{N}-14$} & 1902 & $Q$ & $\mathrm{~B},{ }^{3} \mathrm{H}, \mathrm{NO}_{3}, \gamma$ \\
\hline & & SA & $S r$ \\
\hline \multirow[t]{2}{*}{$N-15$} & 1903 & $Q$ & $\mathrm{~B},{ }^{3} \mathrm{H}, \mathrm{NO}_{3}, \gamma$ \\
\hline & & $S A$ & $\mathrm{Sr}$ \\
\hline
\end{tabular}

300 Area We $11 \mathrm{~s}$

\begin{tabular}{|c|c|c|c|}
\hline$(399-) 1-1$ & 4403 & $Q$ & $\mathrm{~B}, \mathrm{NO}_{3}, \mathrm{U}, \mathrm{Cr}^{+6}, \mathrm{~F}, \gamma$ \\
\hline $1-2$ & 4404 & $Q$ & $\mathrm{~B}, \mathrm{NO}_{3}, \mathrm{U}, \mathrm{Cr}^{+6}, \mathrm{~F}, \mathrm{\gamma}$ \\
\hline $1-3$ & 4406 & $Q$ & $B, \mathrm{NO}_{3}, \mathrm{U}, \mathrm{Cr}^{+6}, \mathrm{~F}, \gamma$ \\
\hline $1-4$ & 4407 & $Q$ & $B, \mathrm{NO}_{3}, U, \mathrm{Cr}^{+6}, \mathrm{~F}, Y$ \\
\hline $1-5$ & 4806 & $M$ & $B, \mathrm{NO}_{3}, U, \mathrm{Cr}^{+6}, \mathrm{~F}, \gamma$ (To HEDL) \\
\hline $1-6$ & 4837 & M & $\mathrm{B}, \mathrm{NO}_{3}, \mathrm{U}, \mathrm{Cr}^{+6}, \mathrm{~F}, \gamma$ \\
\hline $2-1$ & 4402 & $M$ & $\mathrm{~B}, \mathrm{NO}_{3}, \mathrm{U}, \mathrm{Cr}^{+6}, \mathrm{~F}, \mathrm{r}$ \\
\hline $2-2$ & 4633 & M & $\mathrm{B}, \mathrm{NO}_{3}, \mathrm{U}, \mathrm{Cr}^{+6}, \mathrm{~F}, \mathrm{r}$ \\
\hline $2-3$ & 4634 & M & $\mathrm{B}, \mathrm{NO}_{3}, \mathrm{U}, \mathrm{Cr}^{+6}, \mathrm{~F}, \mathrm{r}$ \\
\hline $3-1$ & 4401 & M & $\mathrm{B}, \mathrm{NO}_{3}, \mathrm{U}, \mathrm{Cr}^{+6}, \mathrm{~F}, \mathrm{Y}$ \\
\hline $3-2$ & 3303 & $M$ & $B, \mathrm{NO}_{3}, U, \mathrm{Cr}^{+6}, \mathrm{~F}, \gamma$ \\
\hline $3-3$ & 3034 & M & $\mathrm{E}, \mathrm{NO}_{3}, \mathrm{U}, \mathrm{Cr}^{+6}, \mathrm{~F}, \mathrm{r}$ \\
\hline $3-9$ & 4626 & M & $B, \mathrm{NO}_{3}, U, \mathrm{Cr}^{+6}, F, \gamma, \mathrm{Sr}$ \\
\hline $3-10$ & 4627 & $M$ & $B, \mathrm{NO}_{3}, U, \mathrm{Cr}^{+6}, \mathrm{~F}, \gamma, \mathrm{Sr}$ \\
\hline $3-11$ & 4628 & M & $B, \mathrm{NO}_{3}, \mathrm{U}, \mathrm{Cr}+\epsilon, \mathrm{F}, \gamma, \mathrm{Sr}$ \\
\hline $4-1$ & 4410 & $M$ & $B, \mathrm{NO}_{3}, \mathrm{U}, \mathrm{Cr}^{+6}, \mathrm{~F}, \mathrm{\gamma}$ \\
\hline $4-7$ & 4568 & M & $\mathrm{B}, \mathrm{NO}_{3}, \mathrm{U}, \mathrm{Cr}^{+6}, \mathrm{~F}, \gamma$ \\
\hline $4-9$ & 4629 & M & $B, \mathrm{NO}_{3}, \mathrm{U}, \mathrm{Cr}^{+6}, \mathrm{~F}, Y, \mathrm{Sr}$ \\
\hline $4-10$ & 4630 & M & $B, \mathrm{NO}_{3}, U, \mathrm{Cr}^{+6}, \mathrm{~F}, \gamma, \mathrm{Sr}$ \\
\hline $5-1$ & 4411 & $Q$ & $B, \mathrm{NO}_{3}, U, \mathrm{Cr}^{+6}, \mathrm{~F}, \mathrm{Y}$ \\
\hline $6-1$ & 4409 & $Q$ & $\mathrm{~B}, \mathrm{NO}_{3}, U, \mathrm{Cr}^{+6}, \mathrm{~F}, \gamma$ \\
\hline
\end{tabular}

(a) Water quality analyses (designated WQ) include the following: $\mathrm{pH}$, Conductance, $\mathrm{Ca}, \mathrm{Mg}, \mathrm{Na}, \mathrm{CO}_{3}, \mathrm{HCO}_{3}, \mathrm{~K}, \mathrm{~B}, \mathrm{NO}_{3}-\mathrm{N}, \mathrm{Cl}, \mathrm{SO}_{4}-\mathrm{S}$, and dissolved solids. 
V. GROUND WATER (contd)

\begin{tabular}{|c|c|c|c|}
\hline Well Number & EMA \# & Frequency & Analyses $^{(a)}$ \\
\hline$(399-) 8-1$ & 4405 & Q & $B, \mathrm{NO}_{3}, U, \mathrm{Cr}^{+6}, \mathrm{~F}, \gamma$ \\
\hline $8-2$ & 4408 & Q & $\beta, \mathrm{NO}_{3}, \mathrm{U}, \mathrm{Cr}^{+6}, \mathrm{~F}, \mathrm{\gamma}$ \\
\hline $8-3$ & 4412 & Q & $\mathrm{B}, \mathrm{NO}_{3}, \mathrm{U}, \mathrm{Cr}+6, \mathrm{~F}, \gamma$ \\
\hline $8-4$ & 4865 & M & $\mathrm{B}, \mathrm{NO}_{3}, \mathrm{U}, \mathrm{Cr}^{+6}, \mathrm{~F}, \gamma$ \\
\hline
\end{tabular}

200 Area Wells

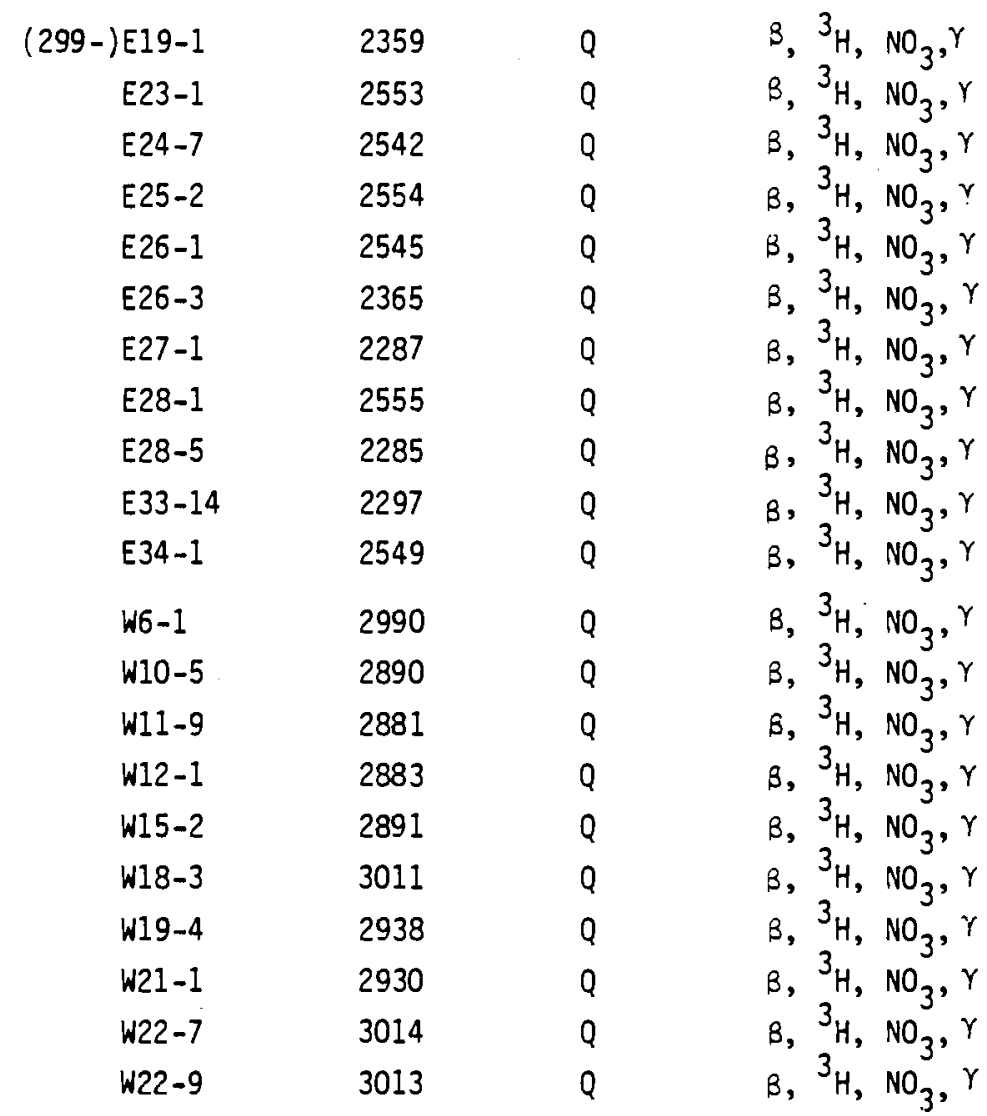

(a) Water quality analyses (designated $W Q$ ) include the following: $\mathrm{pH}$, Conductance, $\mathrm{Ca}, \mathrm{Mg}, \mathrm{Na}, \mathrm{CO}_{3}, \mathrm{HCO}_{3}, \mathrm{~K}, \mathrm{~B}, \mathrm{NO}_{3}-\mathrm{N}, \mathrm{Cl}$, $\mathrm{SO}_{4}-\mathrm{S}$, and dissolved solids. 
VI. FOODSTUFFS

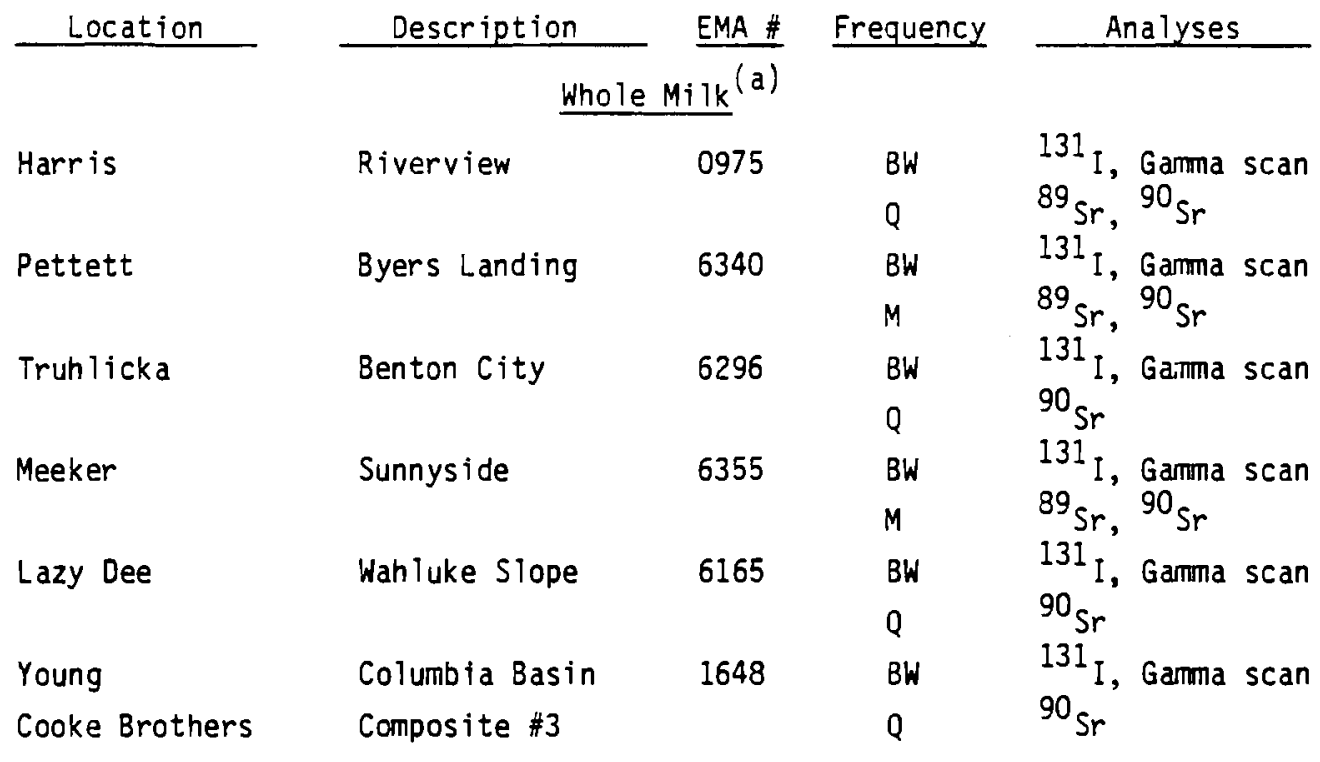

Price

Bleazard

Meat

$\begin{array}{lllll}\text { Harris } & \text { Riverview } & 1292 & \text { SA } & \text { Gamma scan, }{ }^{90} \mathrm{Sr} \\ \text { Pasco Meat Packers } & \text { Commercial } & 1375 & Q & \text { Gamma scan, }{ }^{90} \mathrm{Sr} \\ \text { Local Farm } & \text { FFTF F Sector } & 6418 & \text { A } & \text { Gamma scan }\end{array}$

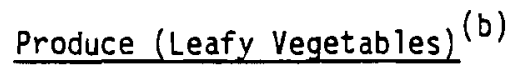

$\begin{array}{lllll}\text { Kinne } & \text { Ringold } & 6373 & \text { A } & \text { Garma scan, }{ }^{90} \mathrm{Sr} \\ \text { Harris } & \text { Riverview } & 1609 & \text { M } & \text { Gamma scan, }{ }^{90} \mathrm{Sr} \\ \text { Local Farm } & \text { Sunnyside } & 6372 & \text { A } & \text { Gamma scan, }{ }^{90} \\ \text { Local Farm } & \text { Benton City } & 1612 & \text { BM } & \text { Garmma scan, }{ }^{\mathrm{Sr}} \\ \text { Local Farm } & \text { Sagemoor Vicinity } & 6419 & \text { A } & \text { Garmma scan } \\ \text { Local Farm (c) } & \text { Walla Walla } & 6409 & \text { M } & \text { Gamma scan } \\ \text { Local Farm (c) } & \text { Moses Lake } & 6407 & \text { M } & \text { Gamma scan } \\ \text { Local Farm (c) } & \text { McNary } & 6410 & \text { M } & \text { Gamma scan } \\ \text { Local Farm (c) } & \text { Othello } & 6408 & \text { M } & \text { Gamma scan }\end{array}$

(a) Sample of forage also collected but not analyzed unless a positive result for 131 I is observed.

(b) Sample collected only during growing season, May to October.

(c) Sample only from one source each month. 
VI. FOODSTUFFS (contd)

\begin{tabular}{|c|c|c|c|c|}
\hline Location & Description & EMA \# & Frequency & Analyses \\
\hline & & Farm Fruit & & \\
\hline Sagemoor Vicinity & Peaches & 0567 & A & Gamma scan \\
\hline Sagemoor Vicinity & Plums & 0577 & A & Gamma scan \\
\hline Sagemoor Vicinity & Grapes & 6417 & A & Gamma scan \\
\hline Sagemoor Vicinity & Apples & 0565 & A & Gamma scan \\
\hline Sagemoor Vicinity & Pears & 6424 & A & Garma scan \\
\hline Sagemoor Vicinity & Cherries & 0543 & A & Gamma scan \\
\hline Sunnyside & Cherries & 6453 & A & Gamma scan \\
\hline Sunnyside & Peaches & 6443 & A & Gamma scan \\
\hline Sunnys ide & Plums & 6442 & A & Gamma scan \\
\hline Sunnyside & Pears & 6441 & A & Garmma scan \\
\hline Sunnyside & App les & 6440 & A & Gamma scan \\
\hline Sunnys ide & Grapes & 6454 & A & Gamma scan \\
\hline
\end{tabular}


VII. WILOLIFE

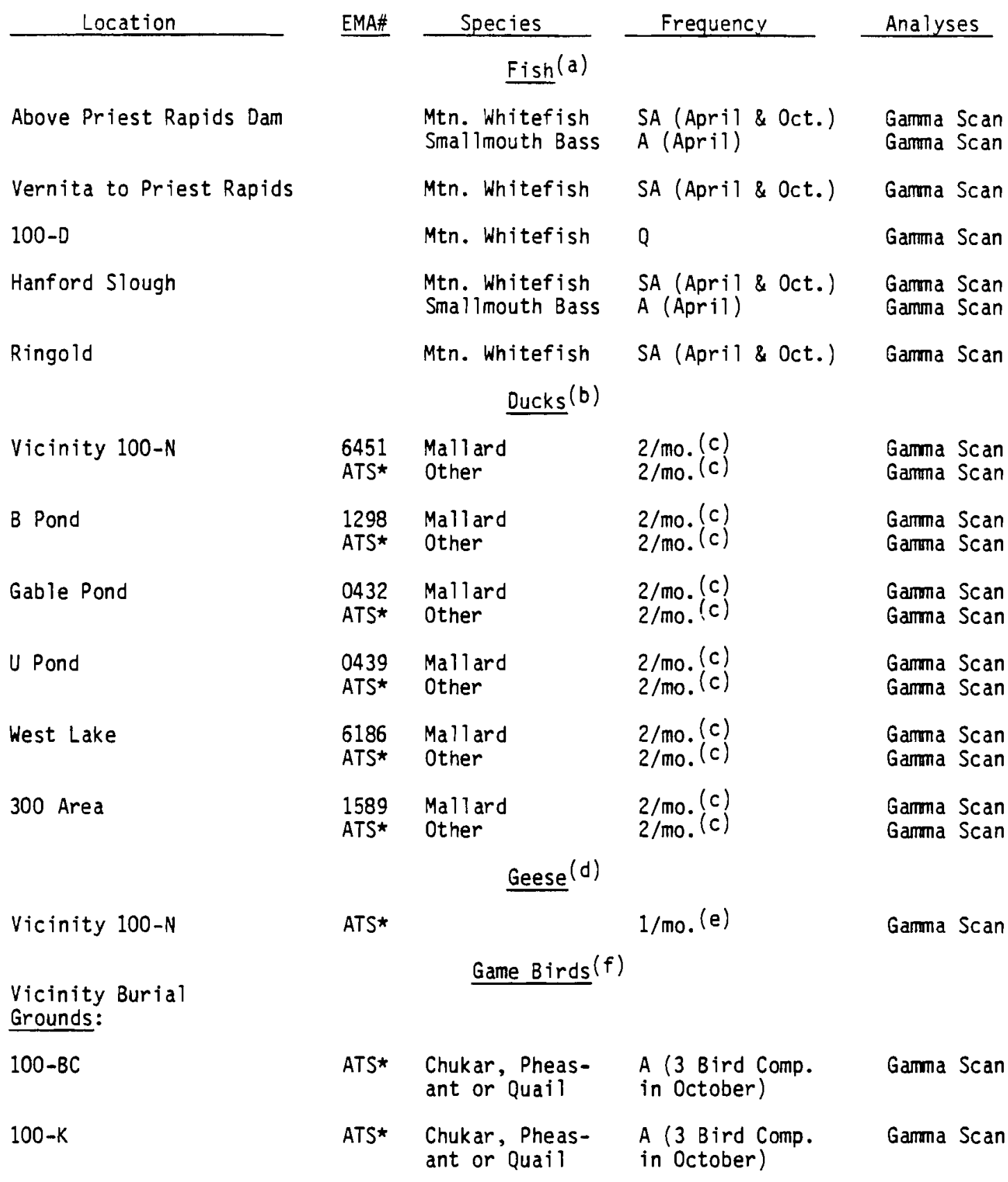

(a) Collect up to five fish at each sampling. Two samples are to be taken from each: 1) skinless muscle fillet from one side of fish and 2) remainder of fish.

(b) Other species such as coot, teal, widgeon, etc., may be used for this sample.

(c) $2 /$ mo. in January, August, and December.

(d) Collect the same species October through December.

(e) $1 /$ mo. in October, November, and December.

(f) Composite the samples only if they are of the same species; otherwise submit as separate samples.

* ATS - according to species. 
VII. WILDLIFE (contd)

\begin{tabular}{|c|c|c|c|c|}
\hline Location & EMA\# & Species & Frequency & Analyses \\
\hline \multicolumn{5}{|c|}{$\underline{\text { Game Birds }}($ contd $)(a)$} \\
\hline $100-n$ & ATS* & $\begin{array}{l}\text { Chukar, Pheas- } \\
\text { ant or Quail }\end{array}$ & $\begin{array}{l}\text { A ( } 3 \text { Bird Comp. } \\
\text { in October) }\end{array}$ & Gamma Scan \\
\hline $100-0$ & ATS* & $\begin{array}{l}\text { Chukar, Pheas- } \\
\text { ant or Quai1 }\end{array}$ & $\begin{array}{l}\text { A (3 Bird Comp. } \\
\text { in October) }\end{array}$ & Garmma Scan \\
\hline $100-H$ & ATS* & $\begin{array}{l}\text { Chukar, Pheas- } \\
\text { ant or Quail }\end{array}$ & $\begin{array}{l}\text { A ( } 3 \text { Bird Comp. } \\
\text { in October) }\end{array}$ & Gamma Scan \\
\hline $100-F$ & ATS* & $\begin{array}{l}\text { Chukar, Pheas- } \\
\text { ant or Quail }\end{array}$ & $\begin{array}{l}\text { A ( } 3 \text { Bird Comp. } \\
\text { in October) }\end{array}$ & Gamma Scan \\
\hline $200 E$ & ATS* & $\begin{array}{l}\text { Chukar, Pheas- } \\
\text { ant or Quail }\end{array}$ & $\begin{array}{l}\text { A ( } 3 \text { Bird Comp. } \\
\text { in October) }\end{array}$ & Gamma Scan \\
\hline $200 \mathrm{~W}$ & ATS* & $\begin{array}{l}\text { Chukar, Pheas- } \\
\text { ant or Quail }\end{array}$ & $\begin{array}{l}\text { A ( } 3 \text { Bird Comp. } \\
\text { in October) }\end{array}$ & Gamma Scan \\
\hline 300 Area & ATS* & $\begin{array}{l}\text { Chukar, Pheas- } \\
\text { ant or Quail }\end{array}$ & $\begin{array}{l}\text { A ( } 3 \text { Bird Comp. } \\
\text { in October) }\end{array}$ & Gamma Scan \\
\hline \multicolumn{5}{|l|}{ OFFSITE } \\
\hline Wahluke Slope & ATS* & $\begin{array}{l}\text { Chukar, Pheas- } \\
\text { ant or Quail }\end{array}$ & $\begin{array}{l}\text { A ( } 3 \text { Bird Comp. } \\
\text { in October) }\end{array}$ & Gamma Scan \\
\hline \multicolumn{5}{|c|}{ Deer } \\
\hline 100 Area & $\begin{array}{l}\text { According } \\
\text { to location }\end{array}$ & Mule & $2 / y r$ & $\begin{array}{l}\text { Muscle - Gamma Scan } \\
\text { Bone - } 90 \mathrm{Sr} \\
\text { Liver - Pu }\end{array}$ \\
\hline 200 Areas & $\begin{array}{l}\text { According } \\
\text { to location }\end{array}$ & Mule & $2 / y r$ & $\begin{array}{l}\text { Muscle - Gamma Scan } \\
\text { Bone - } 90 \mathrm{Sr} \\
\text { Liver - Pu }\end{array}$ \\
\hline 300 Area & $\begin{array}{l}\text { According } \\
\text { to location }\end{array}$ & Mule & $2 / y r$ & $\begin{array}{l}\text { Muscle }-90 \text { Ganma Scan } \\
\text { Bone - } 90 \mathrm{Sr} \\
\text { Liver - Pu }\end{array}$ \\
\hline \multicolumn{5}{|c|}{ Rabbits } \\
\hline 100-N Area & $\begin{array}{l}\text { According } \\
\text { to location }\end{array}$ & Cottontail & $4 / y r$ & $\begin{array}{l}\text { Muscle }-90 \text { Gamma Scan } \\
\text { Bone - } 90 \mathrm{sr}\end{array}$ \\
\hline 200 Areas & $\begin{array}{l}\text { According } \\
\text { to location }\end{array}$ & Cottontail & $4 / y r$ & $\begin{array}{l}\text { Muscle - Ganmia Scan } \\
\text { Bone - } 90 \mathrm{Sr}\end{array}$ \\
\hline 300 Area & $\begin{array}{l}\text { According } \\
\text { to location }\end{array}$ & Cottontail & $4 / y r$ & $\begin{array}{l}\text { Muscle }-90 a m m a ~ S c a n \\
\text { Bone - } 0_{S r}\end{array}$ \\
\hline
\end{tabular}

(a) Composite the samples only if they are of the same species; otherwise submit as separate samples.

* ATS - According to species. 
VIII. SOIL AND VEGETATION

\begin{tabular}{|c|c|c|c|c|}
\hline Location & $\underline{\text { Soil }}$ & Vegetation & Frequency & Analyses \\
\hline Benton City & 6000 & 6046 & A & $\begin{array}{l}\text { Garma scan, } \\
90_{\mathrm{Sr}}, \mathrm{U}, \mathrm{Pu}(\mathrm{a})\end{array}$ \\
\hline Yakima Barricade & 6004 & 6050 & A & $\begin{array}{l}\text { Gamma scan, } \\
90 \mathrm{Sr}, \mathrm{U}, \mathrm{Pu}(\mathrm{a})\end{array}$ \\
\hline Wahluke \#2 Air Sampling Station & 6007 & 6053 & A & $\begin{array}{l}\text { Gamma scan, } \\
{ }^{9}{ }_{S r}, U, P u(a)\end{array}$ \\
\hline Berg Ranch Air Sampling Station & 6008 & 6054 & A & $\begin{array}{l}\text { Gamma scan, } \\
90 \text { Sr, U, Pu }(a)\end{array}$ \\
\hline Ringold & 6009 & 6055 & A & $\begin{array}{l}\text { Gamma scan, } \\
90 \mathrm{Sr}, U, \mathrm{Pu}(\mathrm{a})\end{array}$ \\
\hline $\begin{array}{l}\text { Byers Landing Air Sampling } \\
\text { Station }\end{array}$ & 6011 & 6057 & A & $\begin{array}{l}\text { Garma scan, } \\
90 \text { Sr, U, Pu (a) }\end{array}$ \\
\hline Wye Barricade & 6016 & 6062 & A & $\begin{array}{l}\text { Gamma scan, } \\
9_{\text {Or }}, U, P u(a)\end{array}$ \\
\hline Hanford Townsite (CP\#57) & 6017 & 6063 & A & $\begin{array}{l}\text { Garma scan, } \\
90 \text { Sr, U, Pu }\end{array}$ \\
\hline 200 E Hill Air Sampling Station & 6022 & 6068 & A & $\begin{array}{l}\text { Gamma scan, } \\
90{ }_{S r}, U, P u(a)\end{array}$ \\
\hline Taylor Flats \#2 & 6421 & 6423 & A & $\begin{array}{l}\text { Gamma scan, } \\
9_{S r}, U, P u(a)\end{array}$ \\
\hline Prosser Barricade & 6225 & 6227 & A & $\begin{array}{l}\text { Gamma scan, } \\
90 \mathrm{Sr}, \mathrm{U}, \mathrm{Pu}(\mathrm{a})\end{array}$ \\
\hline East of 200 W Gate (CP\#2) & 6276 & 6283 & A & $\begin{array}{l}\text { Gamma scan, } \\
900_{\text {Sr, U, Pu }}(a)\end{array}$ \\
\hline Southeast Side FFTF site & 6277 & 6286 & A & $\begin{array}{l}\text { Garma scan, } \\
90 \mathrm{Sr}, \mathrm{U}, \mathrm{Pu}(\mathrm{a})\end{array}$ \\
\hline East of ALE Field Lab & 6278 & 6287 & A & $\begin{array}{l}\text { Gamma scan, } \\
90 \mathrm{Sr}, U, \mathrm{Pu}(\mathrm{a})\end{array}$ \\
\hline 1/2 Mile Northeast FFTF Site & 6282 & 6285 & $A$ & $\begin{array}{l}\text { Garma scan, } \\
90 \mathrm{Sr}, \mathrm{U}, \mathrm{Pu}(\mathrm{a})\end{array}$ \\
\hline Sunnyside & 6357 & 6363 & A & $\begin{array}{l}\text { Gamma scan, } \\
9_{S r}, U, P u(a)\end{array}$ \\
\hline Sagemoor Farms & 6358 & 6364 & A & $\begin{array}{l}\text { Gamma scan, } \\
\text { OOS }_{\mathrm{Sr}, U, \mathrm{Pu}}(\mathrm{a})\end{array}$ \\
\hline Taylor Flats \#1 & 6420 & 6422 & A & $\begin{array}{l}\text { Gamma scan, } \\
9_{\text {Sr, U }}, P_{u}(a)\end{array}$ \\
\hline
\end{tabular}

(a) Isotopic Pu. 
VIII. SOIL AND VEGETATION (contd)

\begin{tabular}{lccccc}
\multicolumn{1}{c}{ Location } & Soil & Vegetation & Frequency & Analyses \\
West End Fir Road & 6360 & 6366 & A & $\begin{array}{c}\text { Gamma scan, } \\
90 \text { Sr, U, Pula }\end{array}$ \\
Harris Farm & 6361 & 6367 & A & $\begin{array}{c}\text { Gamma scan, } \\
90 \text { Sr, U, Pu (a) }\end{array}$ \\
200 ENC & 6362 & 6368 & A & $\begin{array}{c}\text { Gamma scan, } \\
90 \text { Sr, U, Pu(a) }\end{array}$
\end{tabular}

(a) Isotopic $\mathrm{Pu}$. 
IX. EXTERNAL RADIATION MEASUREMENT

Thermoluminescent Dosimeters

\begin{tabular}{|c|c|c|c|}
\hline Location & $\underline{E M A \#}$ & Frequency & Measurement \\
\hline Coyote Rapids & 6135 & M & Inmersion Dose \\
\hline Richland Pumphouse & 1715 & M & Inmersion Dose \\
\hline $200 \operatorname{ENC}^{(a)}$ & 1467 & M & Ambient Dose \\
\hline $200 \operatorname{ESE}^{(a)}$ & 1468 & M & Ambient Dose \\
\hline $200 \operatorname{EWC}^{(a)}$ & 1470 & M & Ambient Dose \\
\hline $200 \mathrm{EEC}^{(\mathrm{a})}$ & 1469 & M & Ambient Dose \\
\hline 200 WEC (a) $^{(a)}$ & 1473 & M & Ambient Dose \\
\hline Redox (a) & 1474 & M & Ambient Dose \\
\hline $200 W W C^{(a)}$ & 1471 & M & Ambient Dose \\
\hline 200 WNE $^{(a)}$ & 1472 & M & Ambient Dose \\
\hline 3705 Building & 1486 & M & Ambient Dose \\
\hline ACRMS & 1698 & M & Ambient Dose \\
\hline 300 Pond $(a)$ & 1699 & M & Ambient Dose \\
\hline 300 Southwest Gate ${ }^{(a)}$ & 6163 & $M$ & Ambient Dose \\
\hline 300 South Gate ${ }^{(a)}$ & 6162 & M & Ambient Dose \\
\hline$C P \# 64^{(a)}$ & 6171 & $M$ & Ambient Dose \\
\hline $1100 \operatorname{Area}^{(a)}$ & 6398 & M & Ambient Dose \\
\hline $100-K^{(a)}$ & 1475 & $M$ & Ambient Dose \\
\hline $100-N$ (WPPSS) $^{(a)}$ & 1476 & M & Ambient Dose \\
\hline $100-D^{(a)}$ & 1477 & M & Ambient Dose \\
\hline 100 Area Fire Station (a) & 6164 & M & Ambient Dose \\
\hline $400 E^{(a)}$ & 1729 & M & Ambient Dose \\
\hline $400 w^{(a)}$ & 6468 & M & Ambient Dose \\
\hline $400 s^{(a)}$ & 6469 & M & Ambient Dose \\
\hline $400 N^{(a)}$ & 6470 & M & Ambient Dose \\
\hline FFTF North & 6177 & M & Ambient Dose \\
\hline FFTF Southeast & 6178 & M & Ambient Dose \\
\hline Prosser Barricade ${ }^{(a)}$ & 6176 & $M$ & Anbient Dose \\
\hline Hanford (a) & 1480 & M & Ambient Dose \\
\hline Wye Barricade ${ }^{(a)}$ & 1483 & M & Ambient Dose \\
\hline Rattlesnake Springs(a) & 1485 & M & Ambient Dose \\
\hline $\operatorname{ERC}^{(a)}$ & 1484 & M & Ambient Dose \\
\hline Yakima Barricade $^{(a)}$ & 1482 & M & Ambient Dose \\
\hline Wahluke $\# 2^{(a)}$ & 1490 & M & Ambient Dose \\
\hline
\end{tabular}

(a) Located at Air Sampling Station. 
IX. EXTERNAL RADIATION MEASUREMENT (contd)

\begin{tabular}{|c|c|c|c|}
\hline Location & EMA \# & Frequency & Measurement \\
\hline $\operatorname{Pasco}^{(a)}$ & 1488 & M & Ambient Dose \\
\hline Richland (a) & 1487 & M & Ambient Dose \\
\hline Vernita $(a)$ & 1481 & M & Ambient Dose \\
\hline Benton City(a) & 1499 & $M$ & Ambient Dose \\
\hline Othe $110^{(a)}$ & 1493 & $M$ & Ambient Dose \\
\hline Conne $11^{(a)}$ & 1494 & M & Ambient Dose \\
\hline Berg Ranch ${ }^{(a)}$ & 1491 & M & Ambient Dose \\
\hline Wahluke Wm. (a) & 1495 & M & Ambient Dose \\
\hline Cooke Brothers $(a)$ & 1492 & M & Ambient Dose \\
\hline Byers Landing(a) & 1498 & $M$ & Ambient Dose \\
\hline Pettett $(a)$ & 6353 & $M$ & Ambient Dose \\
\hline Sagemoor Farms & 6354 & $M$ & Ambient Dose \\
\hline Fir Road $(a)$ & 6356 & M & Ambient Dose \\
\hline Walla Walla ${ }^{(a)}$ & 1695 & M & Ambient Dose \\
\hline Sunnyside ${ }^{(a)}$ & 1500 & $M$ & Ambient Dose \\
\hline $\operatorname{McNary}{ }^{(a)}$ & 1696 & M & Ambient Dose \\
\hline Moses Lake ${ }^{(a)}$ & 1693 & M & Ambient Dose \\
\hline Washtucna & 1694 & M & Ambient Dose \\
\hline Up River 100-B Area & 6471 & M & Ambient Dose \\
\hline Below 100-B Retention Basin & 6472 & M & Ambient Dose \\
\hline Above $100-K$ Boat Ramp & 6260 & M & Ambient Dose \\
\hline Below $100-K$ Retention Basins & 6473 & M & Ambient Dose \\
\hline 100-N Trench Springs & 6390 & M & Ambient Dose \\
\hline Down River $100-N$ Trench Springs & 6474 & M & Ambient Dose \\
\hline Down River 100-D & 6475 & M & Ambient Dose \\
\hline Down River Opposite 100-D & 6261 & M & Ambient Dose \\
\hline Lower End Locke Is land & 6262 & M & Ambient Dose \\
\hline White Bluffs Ferry Landing & 6263 & $M$ & Ambient Dose \\
\hline White Bluffs Slough & 6476 & M & Ambient Dose \\
\hline 100-F Area Floodplain & 6477 & $M$ & Ambient Dose \\
\hline Below 100-F & 6264 & M & Ambient Dose \\
\hline Hanford Peninsula & 6478 & M & Ambient Dose \\
\hline Hanford Power line Crossing & 6389 & M & Ambient Dose \\
\hline Hanford Ferry Landing & 6265 & M & Ambient Dose \\
\hline
\end{tabular}

(a) Located at Air Sampling Station. 
IX. EXTERNAL RADIATION MEASUREMENT (contd)

Location
Hanford RR Track
Savage Is I and Slough
Ringold Is land
Powerline Crossing
North End Wooded Island
South End Wooded Island
Island River Mile 344
Is land River Mile 333

\begin{tabular}{|c|c|c|}
\hline EMA \# & Frequency & Measurement \\
\hline 6266 & $M$ & Ambient Dose \\
\hline 6479 & M & Ambient Dose \\
\hline 6267 & M & Ambient Dose \\
\hline 6268 & M & Ambient Dose \\
\hline 6480 & M & Ambient Dose \\
\hline 6269 & M & Ambient Dose \\
\hline 6481 & M & Ambient Dose \\
\hline 6482 & M & Ambient Dose \\
\hline
\end{tabular}

(a) Located at Air Sampling Station. 
X. PORTABLE INSTRUMENT SURVEYS

Columbia River Shoreline

\begin{tabular}{|c|c|c|c|}
\hline Location & $\begin{array}{c}\text { River } \\
\text { Mile }\end{array}$ & EMA \# & Instrument \\
\hline Above 100-K Boat Ramp & $382.5 P$ & 6132 & LLM, GM \\
\hline 100-N Trench Springs & $379.4 P$ & 6129 & LLM, GM \\
\hline Down River Opposite 100-D & $376.8 \mathrm{~F}$ & 6241 & LLM, GM \\
\hline Lower End Locke Is land & $371.0 \mathrm{IP}$ & 6123 & LLM, GM \\
\hline White Bluffs Ferry Landing & $369.7 P$ & 6121 & LLM, GM \\
\hline Below 100-F & $368.3 \mathrm{P}$ & 6120 & LLM, GM \\
\hline Hanford Power line Crossing & $362.6 \mathrm{P}$ & 6118 & LLM, GM \\
\hline Hanford Ferry Landing & $362.0 \mathrm{~F}$ & 6117 & LLM, GM \\
\hline Hanford Railroad Track & $361.4 \mathrm{P}$ & 6242 & LLM, GM \\
\hline Ringold Is land & 354.7 IP & 6114 & LLM, GM \\
\hline Powerline Crossing & $350.4 P$ & 6113 & LLM, GM \\
\hline
\end{tabular}

Roadway Survey

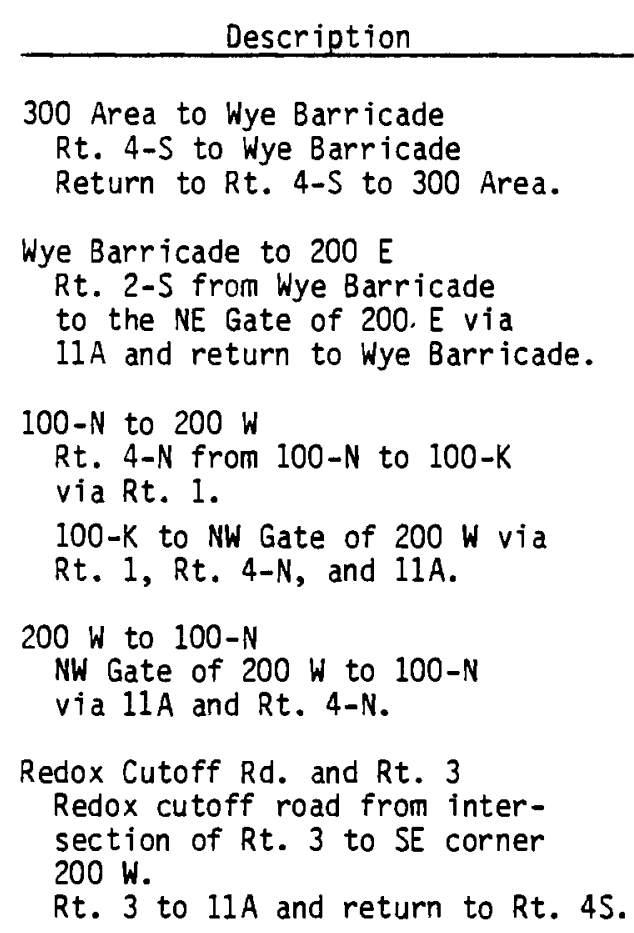

\begin{tabular}{ccc}
$\begin{array}{c}\text { Identification } \\
\text { Number }\end{array}$ & Frequency & Instrument \\
\hline 1 & $M$ & $\begin{array}{c}\text { Bioplastic } \\
\text { Crystal }\end{array}$ \\
2 & $M$ & $\begin{array}{c}\text { Bioplastic } \\
\text { Crystal }\end{array}$ \\
3 & $M$ & $\begin{array}{c}\text { Bioplastic } \\
\text { Crystal }\end{array}$ \\
& & \\
4 & $M$ & Bioplastic \\
& & Crystal \\
5 & $M$ & Bioplastic \\
& & Crystal
\end{tabular}


$X$. PORTABLE INSTRUMENT SURVEYS (contd)

Roadway Survey (contd)

Description

Ident if ication

Number

6

Hanford to $100-\mathrm{N}$

Rt. 2-N from Rt. 11 A to $100-N$ intersection via Rt. 4-N.

100-N intersection to Rt. 11A

via Rt. $4-N$ and Rt. $2-N$.

$11 \mathrm{~A}$ to $100-\mathrm{K}$

$11 \mathrm{~A}$ and Rt. 6 intersection to

$100-K$ via Rt. 6 and Rt. 1 .

Return via Rt. 1 and Rt. 6

to $11 A$.

Yakima Barricade to NECO Entrance

Via $11 \mathrm{~A}$ and Rt. 4-S.

Horn Rapids to $11 \mathrm{~A}$

Rt. 10 from Horn Rapids to

Wye Barricade.

Rt. 4-S from Wye Barricade to 11A.

Railway Survey 300 Area to May Junction
including FFTF Spur

May to Ethe 1

100-N to Nancy to Helen Helen via Audrey to $100-K$, Helen to Susie, Susie to 200 West, Susie to 200 East

100-K to Audrey

Audrey to $100-B$, Audrey to

Vernita Highway

May to Nancy via Ruth and Bettie

Aerial Survey

Project Perimeter
1

2

3

4

5

3
Frequency Instrument

Bioplastic Crystal

Bioplastic Crystal

8

Q

Bioplastic Crystal

9

$M$

Bioplastic Crysta 1 Bioplastic
Crystal

Bioplastic Crystal

Bioplastic Crystal

A

Bioplastic Crystal

A

Bioplastic Crystal

NaI Crystal 
XI. SURVEILLANCE OF WASTE DISPOSAL SITES

Active, inactive, and retired waste disposal sites require periodic monitoring to assure appropriate maintenance. The following sites are routinely checked for vegetation growth, evidence of burrowing animals, erosion, status of enciosure, etc.

Description

Frequency

100-K Trench

A

100-BC SE B.G. (105-C Solid Waste) SA

100-BC SW B.G. (105-B Solid Waste, N. Solid Waste) SA

100-BC Construction B.G. $S A$

100-BC B.G. East of 108-B SA

100-BC Irradiated Metal Storage Basin Waste $S A$

108-B Ball 3x Burial Ground $S A$

108-B Crib SA

105-C Trench SA

105-B Trench SA

107 Basin Sludge Burial SA

105-C Metal Examination Waste Tank SA

100-BC Overflow Pluto Crib SA

107-C Retention Basin SA

$107-B$ Retention Basin SA

100-BC Effluent Diversion Box SA

100-BC Minor B.G.'s East of 105-B SA

100-BC Outfall Structures $S A$

100-DR Outfall Structures SA

100-DDR Trench. SA

107-D Retention Basin SA

107-DR Retention Basin SA

100-DDR Effiuent Lines SA

100-D Durminy Decontamination Waste SA

100-DDR Solid Waste B.G. (VSR Thimbles) SA

100-DDR Construction B.G. SA

100-DDR \#3 B.G. NE of DR SA

100-DDR Pluto Crib SA

100-DDR 105 Basin Sludge B.G. SA

100-DDR \#1 B.G. $S A$

100-DDR \#2 B.G.

100-H Trench SA

107-H Basin SA

100-H Effluent Lines (Junction Boxes) SA 
XI. SURVEILLANCE OF WASTE DISPOSAL SITES (contd)

\begin{tabular}{|c|c|}
\hline Description & Frequency \\
\hline 100-H Liquid Waste Burial & SA \\
\hline $100-H \# 1$ B.G. & SA \\
\hline $100-H$ \#2 B.G. & SA \\
\hline P-11 Area & A \\
\hline 100-F Lewis Canal & SA \\
\hline 100-F Swampy Area & $S A$ \\
\hline 100-F Trench & $S A$ \\
\hline 100-F Retention Basin & SA \\
\hline 100-F Trench Drain and Adjacent Wood Covered Pit & SA \\
\hline 100-F Ball Washer Crib & $S A$ \\
\hline $100-F$ \#3 B.G. & SA \\
\hline 100-F \#2 B.G. & $S A$ \\
\hline $100-F$ \#1 B.G. & SA \\
\hline 100-F Sawdust Burial & SA \\
\hline 100-F Leaching Trench & SA \\
\hline 100-F 60" Overground Pipe & SA \\
\hline 100-F Happy Valley Farm Plots & SA \\
\hline 200 W New Redox Pond (216-S-16) & SA \\
\hline 200 W 01d Redox Pond $(216-5-17)$ & A \\
\hline 200 W U Pond Overflow (216-U-11) & SA \\
\hline 200 E B Pond $(216-B-3)$ & $Q$ \\
\hline 200 E B Pond Ditch \#3 & SA \\
\hline 200 E Purex Crib \#1 $(216-A-6)$ & SA \\
\hline 200 E Purex Crib \#2 $(216-A-30-1)$ & SA \\
\hline $200 \mathrm{E}$ North of Purex Crib \#3 (216-A-37-1) & SA \\
\hline 200 E NE Perimeter Fence & SA \\
\hline 200 E 216-8C Crib Area & SA \\
\hline 200 E California Nuclear B.G. Perimeter & Q \\
\hline 300 Area 300 N B.G. & SA \\
\hline 300 Area 300 Wye B.G. & SA \\
\hline 300 Area \#2 B.G. & SA \\
\hline 300 Area \#3 B.G. & SA \\
\hline 300 Area \#4 8.G. & SA \\
\hline 300 Area \#5 B.G. & SA \\
\hline 300 Area \#7 B.G. & SA \\
\hline 300 West B.G. & SA \\
\hline 300 Area N. Process Trench at Perimeter Fence & $Q$ \\
\hline $200-N, P$, and $R$ Areas & SA \\
\hline
\end{tabular}




\section{DISTRIBUTION}

No. of

Copies

OFFSITE

A. A. Churm

DOE Patent Group

Department of Energy

9800 South Cass Ave.

Argonne, IL 60439

27 DOE Technical Information Center

R. R. Mooney Washington State Department of Social and Health Services

1514 Smith Tower

Seattle, WA 98104

ONSITE

9 DOE Rfefiland Operations Office-

0. J. Elgert

D. R. Elle (5)

H. E. Ransom

M. W. Tiernam

T. E. Aust in

7 Rockwell Hanford Company

W. F. Heine

P. G. Lorenzini

G. C. Owens

J. V. Panesko

W. H. Price

R. E. Wheeler

Rockwell Files
No. of

Copies

Hanford Environmental Health Foundation

B. D. Reinert

3 UNC Nuclear Industries, Inc.

T. E. Dabrowski

E. A. Week $1 y$

UNI Files

2 Westinghouse Hanford Company

P. R. Prevo

R. B. Hall

46 Pacific Northwest Laboratory

G. L. Andersen

L. L. Belt

P. J. B lumer (20)

P. E. Bramson

J. P. Corley

R. L. Dirkes

P. A. Eddy

L. W. Hanke 1

W. M. Harty

J. R. Houston

W. W. King

H. V. Larson

V. L. McGhan

K. R. Price

M. R. Quarders (3)

B. D. Robertson

M. J. Sula

C. M. Unruh

Technical Information (5)

Publishing Coordination GO (2) 
